\title{
Trivium
}

Revue franco-allemande de sciences humaines et sociales - Deutsch-französische Zeitschrift für Geistesund Sozialwissenschaften

$4 \mid 2009$

Les « religions orientales » dans le monde grec et romain

\section{Tradition locale contre unité supra-régionale : le culte de Magna Mater}

\section{Anna-Katharina Rieger}

Traducteur : Anne-Laure Vignaux

\section{OpenEdition}

Journals

Édition électronique

URL : http://journals.openedition.org/trivium/3362

ISSN : 1963-1820

Éditeur

Les éditions de la Maison des sciences de l'Homme

Référence électronique

Anna-Katharina Rieger, «Tradition locale contre unité supra-régionale : le culte de Magna Mater », Trivium [En ligne], 4 | 2009, mis en ligne le 23 octobre 2009, consulté le 07 septembre 2020. URL : http://journals.openedition.org/trivium/3362

Ce document a été généré automatiquement le 7 septembre 2020

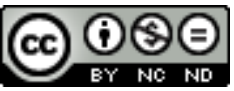

Les contenus des la revue Trivium sont mis à disposition selon les termes de la Licence Creative Commons Attribution - Pas d'Utilisation Commerciale - Pas de Modification 4.0 International. 


\title{
Tradition locale contre unité supra- régionale : le culte de Magna Mater
}

\author{
Anna-Katharina Rieger \\ Traduction : Anne-Laure Vignaux
}

\section{NOTE DE L'ÉDITEUR}

Nous remercions Anna-Katharina Rieger et la maison d'édition Fabrizio Serra pour l'aimable autorisation de publier cet article en traduction française.

\section{NOTE DE L'AUTEUR}

Je remercie Corinne Bonnet, Jörg Rüpke et Paolo Scarpi, les promoteurs de l'accord de recherche trinational « Die orientalischen Religionen in der griechisch-römischen Welt - Les religions orientales dans le monde grec et romain - Le religioni orientale nel mondo greco e romano ", de m'avoir invitée à intervenir lors de leur troisième workshop en mai 2006. Je remercie également Sergio Ribichini et Dirk Steuernagel, pour avoir assuré le suivi rédactionnel de la version écrite.

\section{La transmission du culte de Magna Mater dans le matériel archéologique}

1 Magna Mater, la Grande Mère de l'Asie mineure, constitue un cas intéressant pour l'examen des mécanismes de diffusion des signes religieux. En effet, la transmission de son culte à Rome depuis cette région se prête à une reconstitution historique dans la mesure où suffisamment de traces ont été conservées dans les sources tant écrites qu'archéologiques. De plus, la déesse et sa vénération ont été introduites dans un contexte culturel dont les traditions religieuses étaient différentes de celles de leur 
pays d'origine. Les réactions socio-religieuses déclenchées à l'époque ont été largement commentées, que ce soit dans la littérature antique ou dans la littérature scientifique moderne sur l'Antiquité.

2 Je voudrais retracer à nouveau la route et les moyens de cette transmission vers Rome et, sur cette base, identifier les éléments restés constants lors du transfert et ceux qui ont au contraire subi des changements entre leur apparition à la période hellénistique et l'époque impériale. Il s'agit de voir si Magna Mater a réellement été vénérée partout de la même façon. Son culte s'étant diffusé depuis Rome vers les provinces au début de l'Empire ${ }^{1}$, il faudra également tenter d'identifier les formes locales que le culte a éventuellement revêtues dans les différentes parties de l'Empire. Découlaient-elles des traditions locales liées aux gens et aux régions qui les adoptaient, ou étaient-elles conditionnées par les voies de transmission, les médias ou les personnes qui véhiculaient le culte de la déesse? Les résultats enregistrés sur l'exemple de cette divinité particulière contribueront, en matière d'histoire du culte, à décider de la mesure dans laquelle on peut parler d'unité supra-régionale du culte de Magna Mater dans l'Empire romain ou plutôt de prédominance des traditions cultuelles locales.

3 Je me référerai dans les pages qui suivent aux lieux, aux objets et aux textes du culte de Magna Mater connus à ce jour. Il s'agit de témoignages plus ou moins pertinents de la diffusion et des moyens des pratiques cultuelles. Même si le recensement et l'analyse du matériel resteront nécessairement partiels dans le cadre de cet exposé, l'examen et la confrontation directe de ces sources doivent permettre de donner quelques premières réponses aux questions posées ci-dessus. Je me fonde sur l'architecture sacrée, les objets de culte, les objets votifs et les représentations liés à Magna Mater. Les sanctuaires offrent une première clé d'accès, en tant que lieux physiquement circonscrits où était pratiqué le culte. Leur situation, leur conception et leur architecture donnent une idée de la vie qui s'y déroulait et permettent de reconnaître des particularités éventuelles de la divinité et de son culte. L'inventaire des sanctuaires - matériel votif, statues, inscriptions - offre comme on sait des possibilités d'analyse du cercle des personnes impliquées dans le culte et des formes de représentation de ces dernières. La statuaire - ses motifs et son iconographie - permet à son tour de comprendre l'organisation locale du culte de Magna Mater et peut, tout comme les voies et les sphères d'action des adorateurs et des spécialistes religieux, faire apparaître des liens avec les traditions propres à d'autres lieux de culte.

Car on ignore encore, par exemple, comment on avait appris, en Gaule, l'existence du sanctuaire romain de Magna Mater in agro Vaticano ou ce qu'un prêtre de la déesse pouvait divulguer sur son chemin entre Carthage et Rome. Ou encore, sur quels critères on choisissait les formes architecturales et l'agencement d'un sanctuaire de Magna Mater à Rome, dans le Latium ou en Asie mineure.

5 Nos réflexions commencent à Rome, dans le sanctuaire de Magna Mater du Palatin, car c'est officiellement là que la délégation chargée du transport de la déesse depuis l'Asie mineure foula pour la première fois le sol de l'Empire (fig. 1). Il reste encore à expliquer ce qui fut au juste introduit au cœur de la cité et repris d'Asie mineure. 


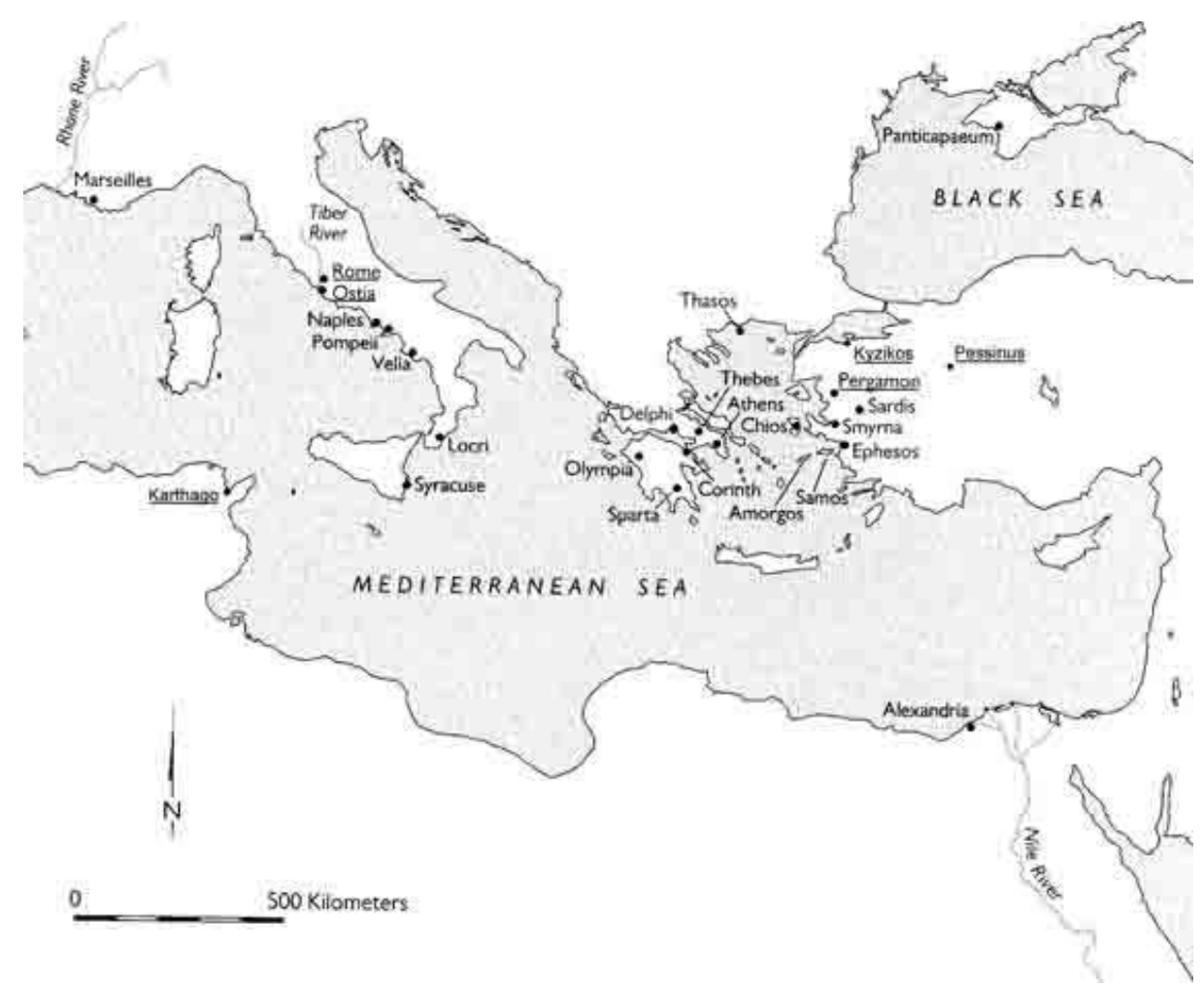

Fig. 1. Carte de la Méditerranée avec quelques lieux importants pour le culte de Magna Mater. Roller (1999). Carte 2 avec changements de l'auteur.

Cependant, l'arrivée à Rome fut nécessairement précédée de la remontée du Tibre. Ostie doit en effet être considérée comme le lieu où le navire transportant Magna Mater arriva concrètement, avant d'ensuite emprunter le fleuve. Bien que Rome et Ostie aient eu des relations très étroites, notamment en ce qui concerne les affaires religieuses, la confrontation de ces lieux montre que seuls quelques éléments du culte restèrent inchangés et que, dans le domaine de certaines pratiques, une tradition cultuelle locale se développa dans la ville portuaire malgré la proximité de l'Urbs ${ }^{2}$.

Il fallut attendre l'Empire pour que le culte de Magna Mater quitte Rome, le centre de l'Empire, et essaime vers les villes de province. Ce processus conduit tout naturellement à se demander si ce sont des copies conformes du culte romain de Magna Mater ou bien des pratiques indépendantes qui s'y développèrent.

\section{Magna Mater à Rome}

\subsection{L'arrivée à Rome : lieux, symboles et personnes}

8 À la différence notamment des dieux égyptiens, Magna Mater n'est en fait pas une inconnue à Rome : comme d'autres divinités, elle y est amenée sur ordre des livres sibyllins et par l'entremise de fonctionnaires politico-religieux de haut rang ${ }^{3}$. Elle est par là-même admise dans le canon des cultes officiels, qui supposent l'accomplissement d'un sacrifice par le praetor urbanus et une implication de la haute société locale. Plus tard, son culte sera lié de façon particulièrement intime aux nouveaux tenants du pouvoir puisque la vénération de l'empereur recevra une place particulière dans le culte de Magna Mater. Malgré cela, Magna Mater est encore classée dans certaines 
publications parmi les divinités « orientales » et décrite comme une «étrangère » au culte « quasi-officiel ${ }^{4} »$. Pourquoi?

Ce que l'on adopte à Rome en 204 av. J.-C. n'est pas seulement un nouveau culte publique qui pourra être incorporé sans problèmes et ne représente donc rien de vraiment neuf. Il est en effet accompagné d'autres divinités et de pratiques cultuelles peu connues dans la Rome de la période républicaine ou incompatibles avec les conceptions religieuses et morales, mais aussi légales de la société en place. Ainsi, le héros Attis, qui accompagne Magna Mater à Rome et qui selon la tradition mythologique s'émascule pour elle, n'est-il pas encore un élément fixe de sa vénération à l'époque hellénistique républicaine. En revanche, les prêtres de Magna Mater, ou Galli, doivent être eunuques pour pouvoir entrer au service de la déesse - une condition qui, dans la Rome républicaine au moins, entraînait pour la personne concernée une dégradation légale et des sanctions sociales.

En dépit de ces éléments cultuels étranges pour l'époque, Magna Mater ne tarde pas à devenir dans l'Urbs une déesse aimée - un processus probablement favorisé par le fait que dès avant l'époque de son introduction, elle est vénérée dans les régions grecques et italiques sous sa forme gréco-classique de déesse sur un trône flanqué de lions, avec tympan et couronne murale (fig. 2$)^{5}$.

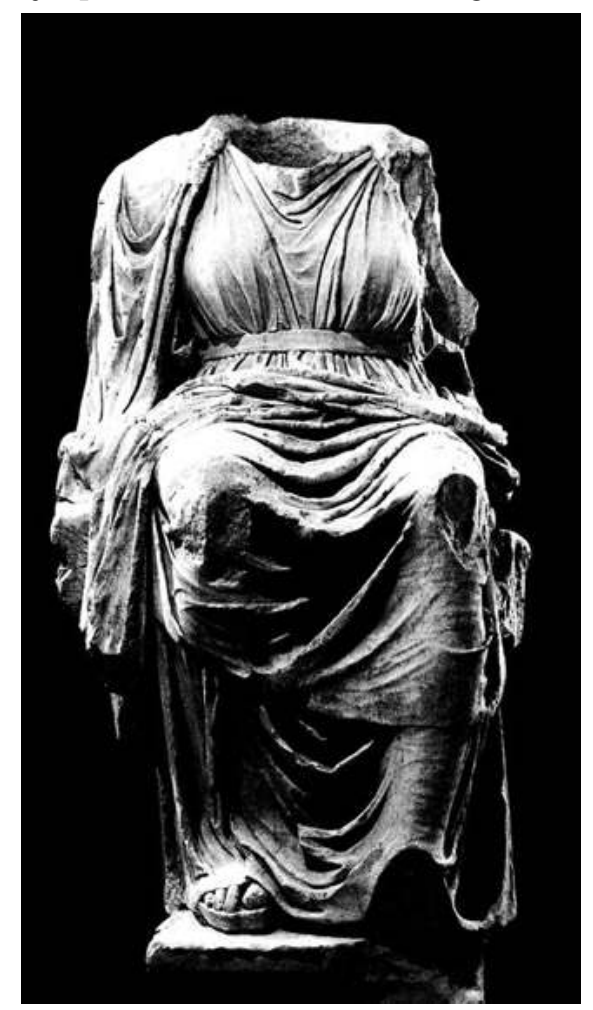

Fig. 2. Statue de Magna Mater du type de l'Agorakritos de Paros, temple de Magna Mater au Palatin, Rome. Musée du Palatin (milieu du ॥e siècle ap. J.-C.). Tomei (1997), p. 148, cat. 128.

Ce n'est pas à une inconnue ou à une étrangère que la société romaine de la fin du III siècle est confrontée. Seule la reprise concrète et politiquement motivée de son culte venu d'Asie mineure exige un examen détaillé et adapté à ce cadre d'analyse. J'aimerais commencer par la question de ce qui a été introduit et à partir d'où. L'objet qui arrive à Rome est la pierre noire, le symbole cultuel aniconique de la Magna Mater d'Asie mineure ${ }^{6}$. En revanche, son lieu de départ ne fait pas l'unanimité. Les versions 
concurrentes, qui proposent soit la Pergame de Mysie, soit la Pessinonte de Phrygie ou de Galatie, trahissent chacune l'arrière-plan politico-religieux de l'introduction de Magna Mater en termes de stratégie politique intérieure ou extérieure des classes dirigeantes romaines (fig. 1) ${ }^{7}$. Les deux lieux sont corrects dans la perspective de leurs auteurs respectifs, car ce ne sont pas les voies exactes de transmission ou les traditions cultuelles historiquement prouvées qui jouent ici le rôle déterminant, mais bien une falsification socialement opportune de l'histoire ${ }^{8}$. Sans doute est-ce en combinant ces deux lieux de provenance que l'on sera le plus proche de la vérité : l'objet cultuel qui symbolise la déesse semble provenir de Pessinonte, centre de la vénération de Cybèle en Asie mineure, qui reçut d'ailleurs ce statut pour la première fois à la période hellénistique, sous l'influence des Attalides'. Quant à Pergame, elle constitue l'intermédiaire politiquement important de cette mission, car ses rois arrangèrent le transfert du culte entre Pessinonte et Rome ${ }^{10}$. C'est aussi de cette ville que le bateau transportant Magna Mater à son bord prit finalement la mer pour Rome.

11 Un traitement similaire des localités, fondé sur les mêmes motivations, s'observe dans le choix des épithètes qualifiant Magna Mater. À Rome, elle est souvent nommée Magna Mater Deum Idaea, en référence au mont Ida, près de Troie, où elle vit. Comme, à partir de l'Empire, Rome et les Romains revendiquent Troie comme ville mère et le héros troyen Énée, dont la divinité protectrice est Magna Mater, comme ancêtre mythique, ils sont tout disposés à reprendre ce nom sans que cela représente à leurs yeux une contradiction par rapport à ses origines pessinontiennes ${ }^{11}$. Dès cette étape précoce de la transmission du culte, on constate donc que, dans la société romaine, une chose nous apparaissant aujourd'hui aussi indiscutable que le lieu d'origine d'une divinité est variable et soutient opportunément la réalisation d'objectifs politico-historiques.

Nous pouvons à présent nous demander quelles personnes accompagnent Magna Mater ou le symbole cultuel depuis sa patrie jusqu'à Rome. Selon les sources, un prêtre et une prêtresse - venant probablement du sanctuaire de Magna Mater à Pergame, ou Megalesium - accompagnent la pierre noire ${ }^{12}$. Deux spécialistes religieux font donc leur entrée à Rome avec l'objet cultuel et restent à son service dans le nouveau lieu de culte. Rappelons en ce qui concerne le processus de transmission qui nous intéresse que la phase d'introduction de Magna Mater est le seul moment connu où les Phrygiens et des personnes originaires d'Asie mineure sont impliqués dans la transmission du culte. C'est l'unique cas où l'origine de Magna Mater joue un rôle important dans la diffusion compétente et efficace de sa vénération - on n'en trouve aucun autre par la suite, du moins si l'on en juge par les témoignages et le matériel conservés.

Dans leur fonction de prêtre, ces personnes apportent naturellement avec elles vêtements et accessoires de culte, qui deviennent dès lors visibles et connus à Rome. Mais les maigres sources dont nous disposons ne permettent guère de se faire une idée exacte de l'apparence des premiers prêtres romains de Magna Mater. La seule question de savoir quel cercle de personnes eut l'autorisation légale de reprendre la fonction de prêtre à l'époque républicaine, à la suite des premiers prêtres d'Asie mineure, pose déjà problème $^{13}$. La description d'un prêtre de Pessinonte ayant passé quelque temps à Rome constitue l'unique source républicaine susceptible de décrire l'apparence d'un prêtre étranger de Magna Mater. Son riche habit sacerdotal, composé d'un manteau brodé d'or et d'une corona, exalte les esprits et, de ce fait, est jugée digne de mention ${ }^{14}$. Le fait qu'aucune représentation iconographique $d u$ culte dans ses premiers siècles d'existence n'ait été conservée à Rome tient probablement à l'apparition réglementée 
des prêtres en public et au peu d'intérêt qu'ils accordaient aux représentations. Si les premières représentations connues de Galli, ou sacerdotes Matris Deum datent de l'Empire, c'est sans doute parce qu'à la suite de l'introduction de nouvelles formes de vénération, le cercle des participants au culte de Magna Mater n'était plus limité aux patriciens, et, qu'en général, l'intérêt pour l'exotisme des divinités augmenta sous l'Empire $^{15}$. Ces images de prêtres sont donc les premières à illustrer des attributs vestimentaires et des objets de culte de Magna Mater inhabituels à Rome.

Les détails iconographiques des rares exemples conservés ne permettent pas de déduire une évolution substantielle de l'image des prêtres de Magna Mater. Mais on peut observer que l'origine étrangère du culte - qu'elle soit à prendre au sens propre ou seulement comme une sorte d'insigne - apparaît dans les représentations et que les éléments visiblement non romains de leur habit - occabus, torques, corona et anaxyrides sont considérés jusque tard dans l'Empire comme des insignes de leur fonction, ainsi que nous l'apprennent deux inscriptions ${ }^{16}$.

L'une des œuvres sculptées les plus anciennes est le relief de Laberia Felicla, de Rome ( ${ }^{\text {er }}$ siècle ap. J.-C.), qui montre la prêtresse vêtue d'un chiton et d'un himation, en train d'effectuer une libation. Outre l'inscription, qui la désigne comme sacerdos maxima matris deum m(agnae) I(daeaa), seul le pectoral, montrant un buste barbu, renvoie à son activité au service de Magna Mater ${ }^{17}$. Nous possédons une image de prêtre nettement plus détaillée sur un relief funéraire des environs de Rome (milieu du II siècle ap. J.-C.). On le voit vêtu d'un habit composé d'un pectoral historié et d'une parure de tête avec plaquettes de buste, entouré d'une série d'instruments de culte tels cymbala, pedum, cista, aspergillum et flagellum (fig. 3).

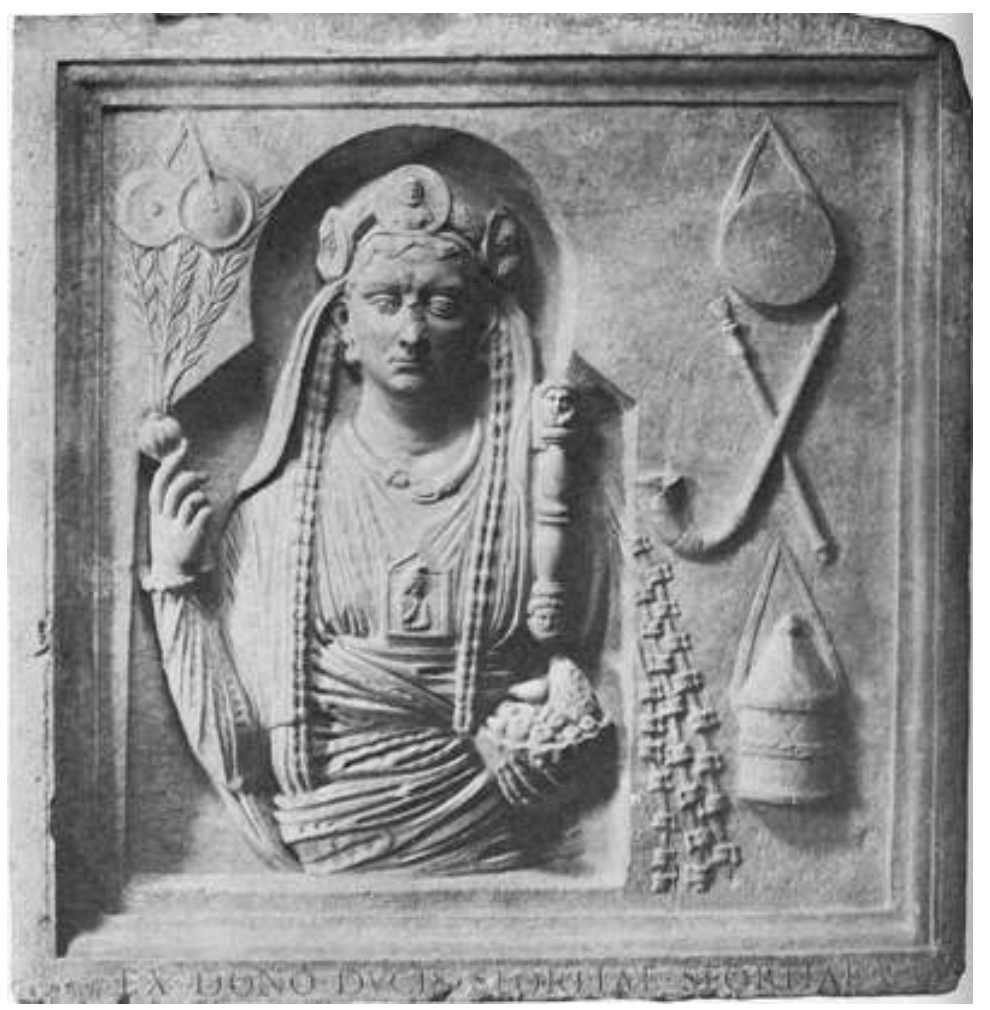

Fig. 3. Relief funéraire d'un prêtre de Magna Mater (gallus) provenant de Lavinium. Rome, Musées capitolins (milieu du ॥e siècle ap. J.-C.). CCCA, III, 1977, n 466, pl. 296. 
16 J.-C.), qui montre un gallus juvénile officiant en vêtements grecs rehaussés de quelques
éléments décoratifs, dont des torques et un grand pectoral en forme de naikos (fig. 4).

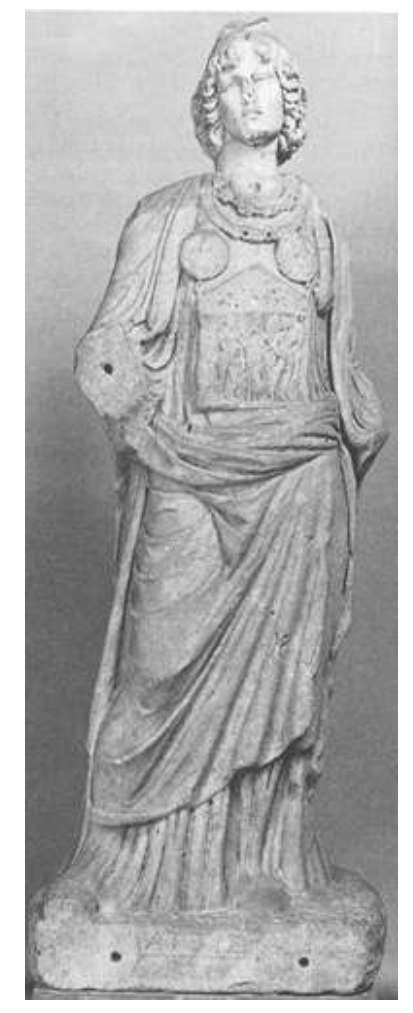

Fig. 4. Statue d'un prêtre de Magna Mater (gallus) provenant de Rome. Rome, Musées capitolins (fin du ॥e siècle ap. J.-C.). Roller (1999), fig. 70.

Seul son bonnet phrygien constitue une caractéristique vestimentaire propre à l'Asie mineure. Il n'est plus possible de voir si, à l'instar de la figure représentée sur le relief, il était vêtu d'une tunica manicata, élément lui aussi inhabituel en Occident à cette époque. On trouve aussi, dans les nécropoles d'Ostie cette fois, des reliefs et un sarcophage (fig. 5 et 6 ) (seconde moitié du III ${ }^{\mathrm{e}}$ siècle ap. J.-C.) montrant un prêtre en habit sacerdotal, avec occabus, pectoral et couronne. 


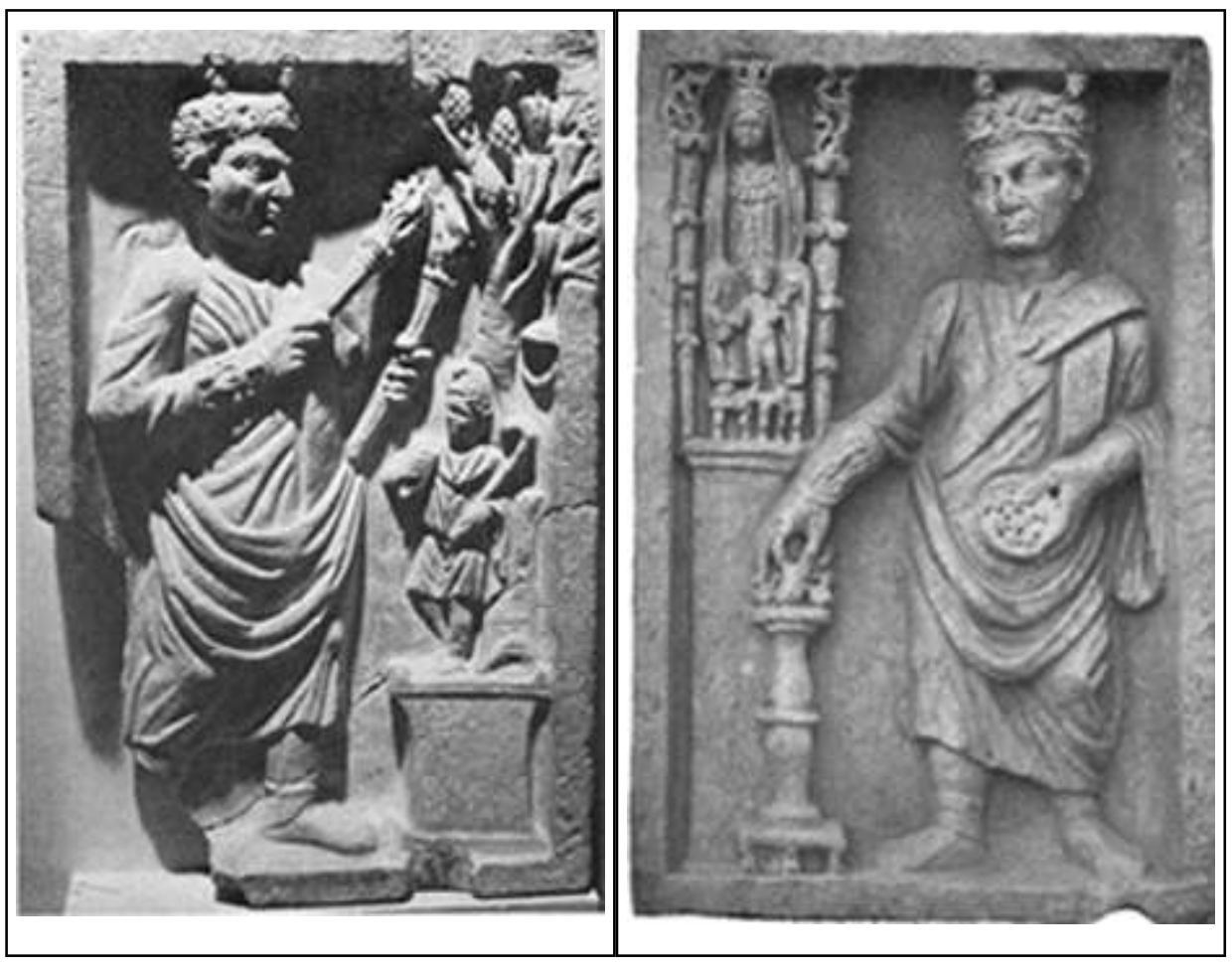

Fig 5 a. b. Reliefs d'un monument funéraire de l'Isola sacra (Ostie) avec représentation d'un prêtre de Magna Mater (gallus). Ostie, Antiquarium (milieu du ॥” siècle ap. J.-C.). CCCA, III, 1977, n² 447-448, pl. 284, 287.

Les torques, qui, dans les représentations, caractérisaient ses prédécesseurs en tant que non-Romains, font ici défaut. Son vêtement ne diffère pas du costume traditionnel romain contemporain, mais il porte en dessous l'anaxyrides d'Asie mineure ${ }^{18}$.

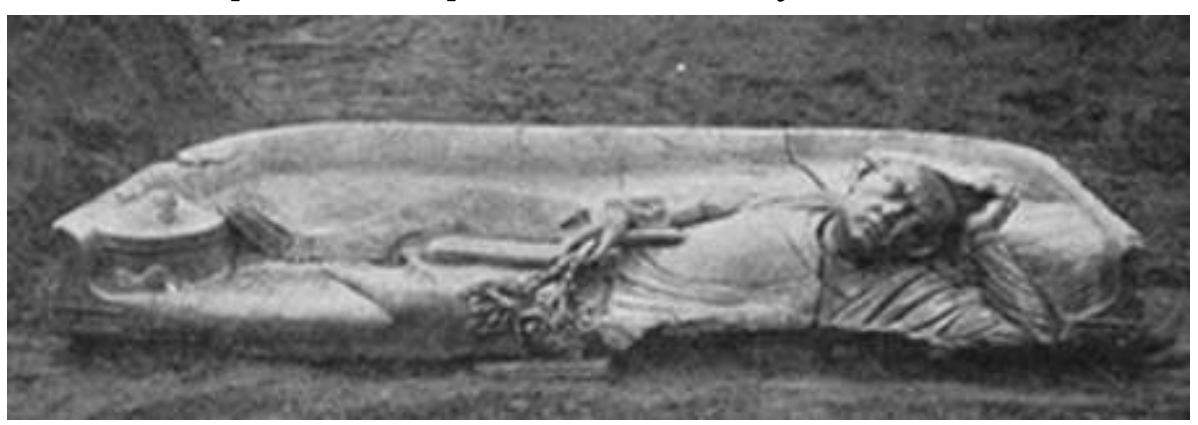

Fig. 6. Sarcophage en forme de kline figurant un prêtre de Magna Mater (gallus) de l'Isola sacra (Ostie). Ostie, Antiquarium (milieu du III siècle ap. J.-C.). Guido Calza, Ostia. - Isola sacra. - Le necropoli del «Portus Romae », « Notizie degli scavi di Antichità », 1931, p. 510-542, fig. I, p. 512.

17 Les lieux de découverte des œuvres citées donnent également des indications sur les mécanismes de diffusion des signes religieux: toutes les représentations de Galli montrées viennent soit de l'Urbs, soit du Latium. Seules deux inscriptions nous montrent que certains éléments vestimentaires étaient également portés en Campanie et en Gaule ${ }^{19}$. Selon moi, il ne faut pas considérer cette distribution géographique comme un hasard de la conservation des sources, mais plutôt comme le fruit probable des différentes capacités régionales à adopter le costume complet des prêtres d'Asie mineure: les symboles du culte de Magna Mater étaient présents à Rome depuis plusieurs siècles et de manière continue. Vu la proximité de Rome, ainsi que la présence et la visibilité des prêtres étrangers, les traditions, l'habit et les autres 
insignes matériels qu'ils avaient amenés à Rome avaient de meilleures chances d'être repris dans les régions limitrophes. Ils représentent donc un élément cultuel régionalement limité.

\subsection{Les éléments authentiquement romains de la vénération de Magna Mater}

\subsubsection{Lieu de culte et fête}

18 Le choix du lieu de vénération de Magna Mater est lié au contexte politique de l'introduction de son culte : la déesse troyenne sera vénérée sur le Palatin, à proximité de la Roma quadrata, du Lupercal et de la hutte de Romulus - un lieu central de la cité, non seulement d'un point de vue spatial, mais aussi d'un point de vue mythique et historique ${ }^{20}$. Parallèlement, le Palatin est à l'époque républicaine le quartier résidentiel des représentants les plus influents de la communauté romaine, eux-mêmes impliqués dans l'introduction du culte, et sera plus tard agrandi pour abriter la demeure de l'empereur. La terrasse du temple de Magna Mater servira de référence à toutes les dispositions architecturales futures.

La situation, mais aussi l'architecture du lieu de culte de Magna Mater correspondent tout à fait à la tradition romaine et ne renvoient en rien aux origines asiatiques de la déesse (fig. 7-9). Après un hébergement provisoire de son symbole cultuel dans le temple de la Victoire, dans le sud-ouest du Palatin, un sanctuaire neuf lui est consacré à proximité en 191 av. J.-C.

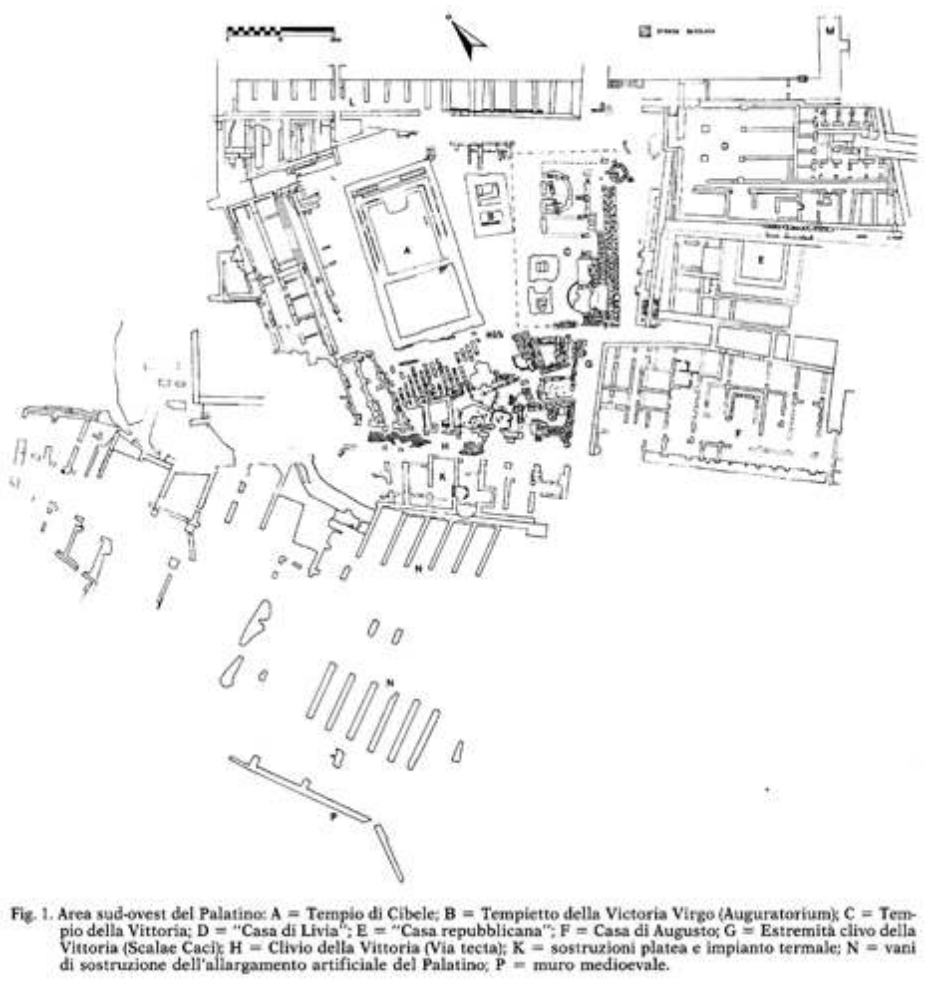

Fig. 7. Reconstitution du plan du temple de Magna Mater sur le Palatin à Rome. Pensabene (1988) ; fig. 1.

Celui-ci se présente comme un temple à podium, tel que l'on en construit partout dans la ville à l'époque; son unique particularité se situe dans la hauteur colossale de son 
soubassement. Conditionné par ce haut socle, le temple possède en guise d'accès un important escalier qui sert aussi de tribune lors des festivités organisées dans l'avantcour (fig. 8-9) ${ }^{21}$.

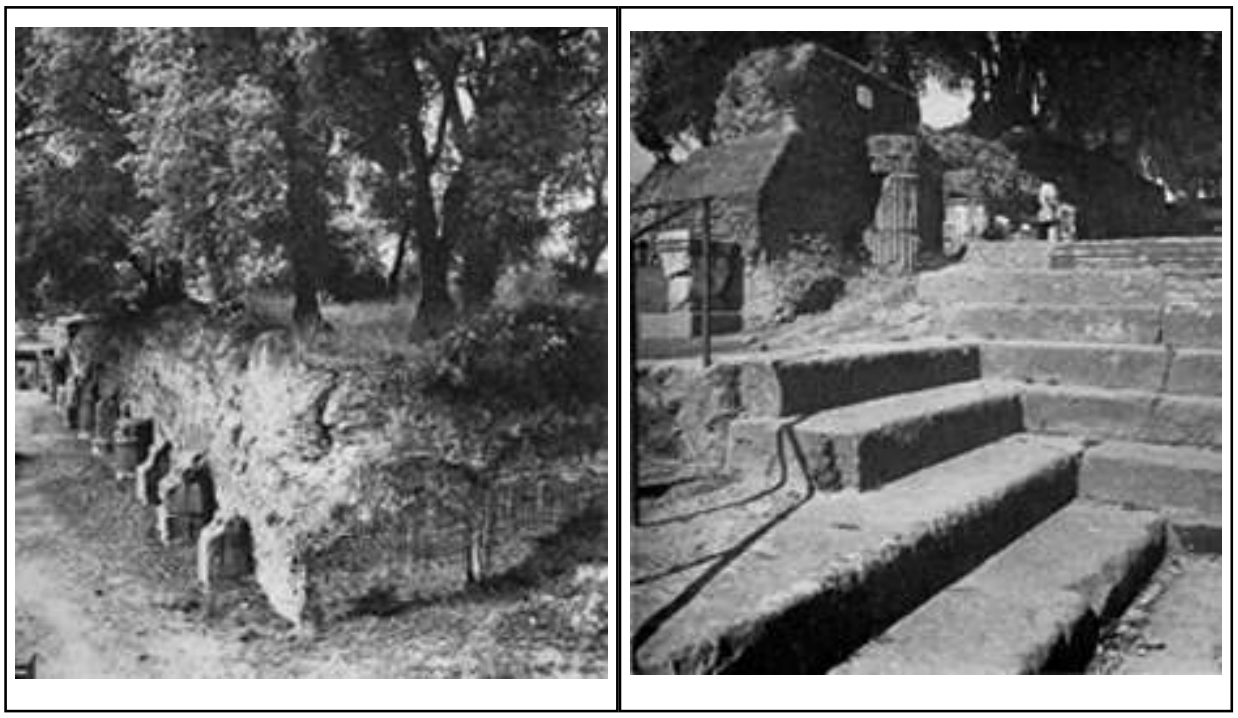

Fig. 8. a. b. Vues du podium depuis l'est et des escaliers précédant le temple de Magna Mater sur le Palatin à Rome. CCCA, III, 1977, pl. 4, 5.

À la suite des changements affectant le déroulement des fêtes et l'affluence de visiteurs, cette cour ne tarda pas à être jugée trop exiguë puis agrandie, tant à l'époque républicaine que sous l'Empire, par le biais de substructions ${ }^{22}$.

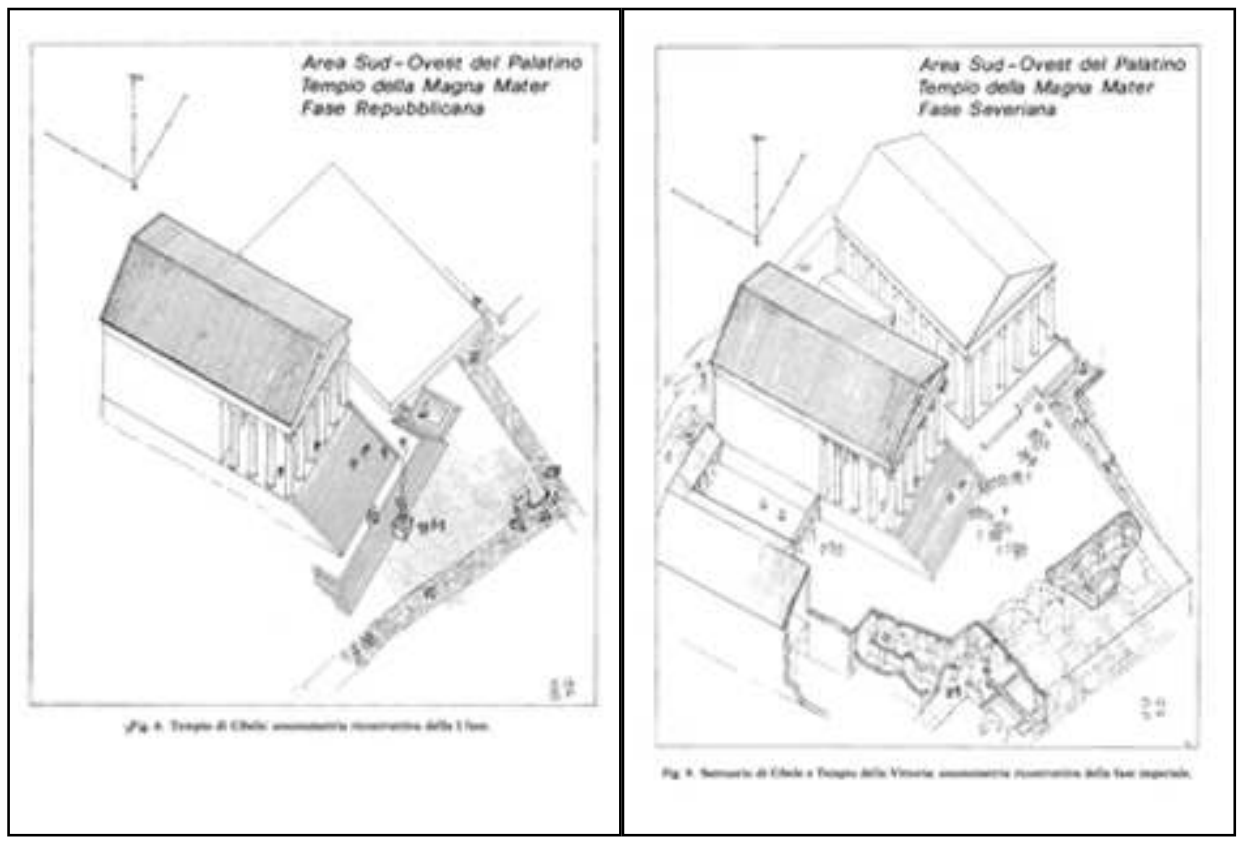

Fig. 9 a. b. Reconstructions du temple de Magna Mater sur le Palatin, avec l'avant-cour et les escaliers-tribune de l'époque républicaine et sévère. Pensabene (1988), fig. 6, 9.

L'architecture des lieux de culte n'apparait en aucun cas étrangère. On pourra certes tenter de trouver dans les proportions et l'agencement intérieur du temple palatin des 
caractéristiques propres à l'architecture des temples d'Asie mineure, mais il s'agira tout au plus de l'affaire d'adeptes de la comparaison architecturale minutieuse. Une telle entreprise ne révèle en tout cas aucun rapport clair avec les temples de la région d'origine de Magna Mater ${ }^{23}$. L'absence à Rome de sanctuaires rupestres ou d'aires ouvertes consacrés suivant les exemples asiatiques et subitaliques, à Magna Mater montre que seuls certains éléments de la vénération de la déesse furent repris d'Asie mineure et combien cette reprise fut sélective ${ }^{24}$.

On crée donc un lieu de culte romain conforme aux attentes de la société romaine qui adopte ce culte et aux réalités rituelles et architecturales locales. Les prêtres d'Asie mineure ne semblent pas non plus avoir vu dans la nature du lieu un problème, ce qui permet de déduire que les pratiques cultuelles reprises présentaient un caractère clairement hellénisé.

Comme le temple, la forme des fêtes données en honneur de Magna Mater correspondait aux habitudes cultuelles romaines. Les Megalesia - dont le nom grec dérive sans doute de Megalesium, mot désignant le sanctuaire de Magna Mater à Pergame - sont organisées à la date anniversaire de l'introduction de la mère des dieux, en avril. Elles s'accompagnent de jeux, de concours et de représentations dramatiques (ludi Megalenses) qui se déroulaient selon les sources antiques à proximité immédiate du temple, sur l'escalier transformé pour l'occasion en tribune. Ces manifestations en l'honneur d'une divinité sont traditionnelles à Rome. Des Ludi sont également célébrés pour quelques autres dieux comme Jupiter, Apollon ou les Dioscures ${ }^{25}$. Autre élément totalement inconnu en Asie mineure, les mutitationes cenarum, également organisées lors des Megalesia, appartiennent aux habitudes religieuses des patriciens romains. Elles consistent à s'inviter mutuellement en l'honneur de la déesse et à prendre des repas en commun.

Des composantes cultuelles connues à Rome et dans la pratique rituelle romaine sont remises à l'honneur pour Magna Mater, si bien que non contente d'être rendue socialement compatible grâce à la forme architecturale de son temple, sa vénération va jusqu'à renforcer les liens sociaux dans la haute société. Tant le lieu de culte de la déesse que les pratiques cultuelles choisies pour elle à Rome sont donc dans une large mesure influencées et transformées par la société d'adoption.

\subsubsection{Attis, accompagnateur de Magna Mater, comme particularité romaine de son culte}

La reprise d'un numen coniunctum de Magna Mater n'est attestée à Rome que dans l'iconographie. Le matériel de fouille du temple du Palatin, que l'on date du $\mathrm{II}^{\mathrm{e}}$ et du $\mathrm{I}^{\mathrm{er}}$ siècle av. J.-C., compte de nombreuses terres cuites votives de petite taille dont les représentations vont, au-delà de la Magna Mater au lion, des animaux sacrificiels aux parties de corps, en passant par les pommes de pin et les figurines d'Attis. Ces dernières apparaissent non seulement dans une grande diversité de types, mais aussi dix fois plus souvent que les représentations de Magna Mater, déesse principale du lieu (fig. 10) ${ }^{26}$.

Fig. 10 a. b. Figurines en terre cuite liées au culte d'Attis sur le Palatin. 


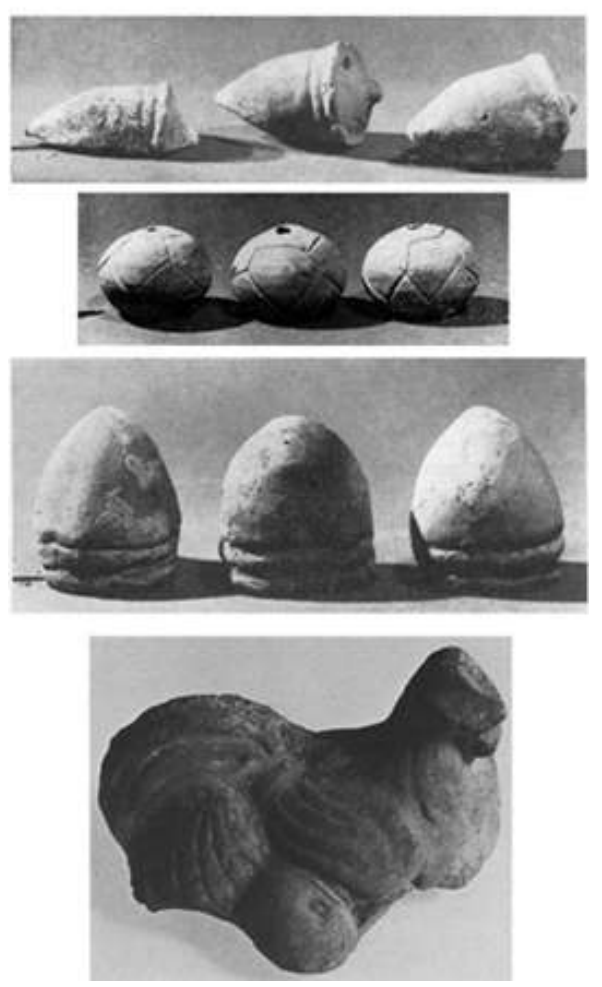

Fig. 10 a Pensabene (1982), pl. 6, 4 ; Romanelli (1963), fig. 62, 63, p. 290.
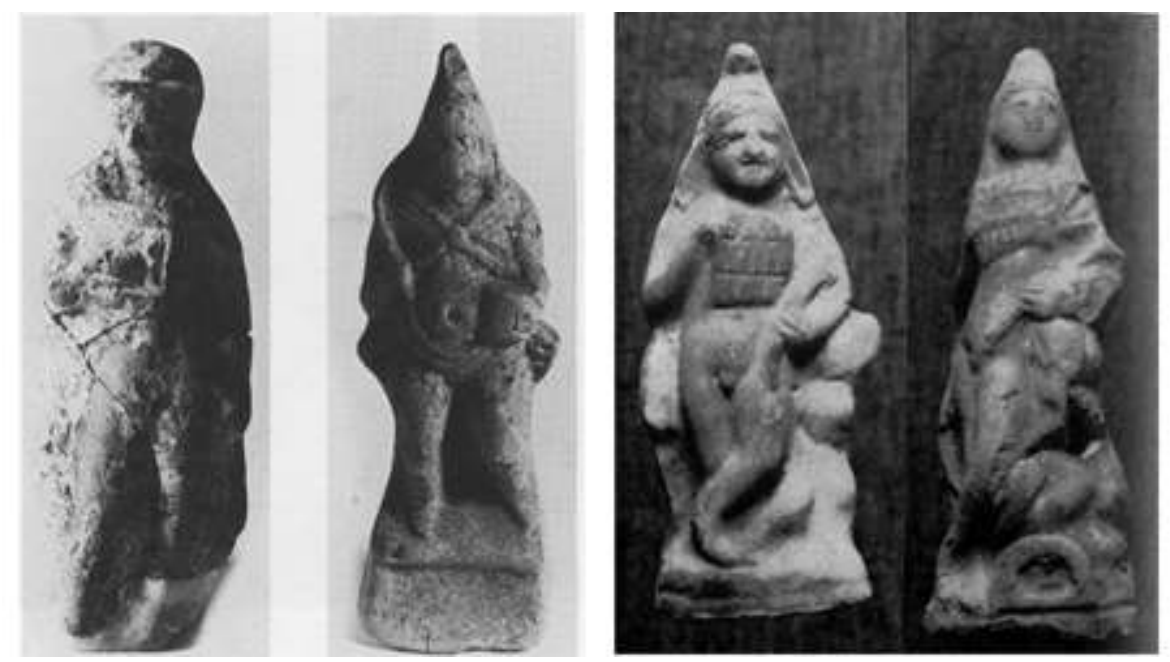

Fig. 10 b Pensabene (1982), pl. 6, 1-2 ; Romanelli (1963), fig. 55, 56, p. 283 sq.

Bien que ne représentant en elles-mêmes qu'un matériel de série assez insignifiant, ces figurines constituent sur le Palatin de la période républicaine une particularité. D'une part, Attis n'est mentionné nulle part dans les sources épigraphiques relatives à l'introduction de Magna Mater, d'autre part, sa vénération n'est d'une manière générale pas très répandue à la période hellénistique. On sait qu'il fait l'objet de pratiques rituelles à Athènes et au Pirée, à Myrina et à Amphipolis - dans des villes de la mère patrie grecque, donc - ainsi qu'à Tarente et à Rhegium en Italie du Sud ${ }^{27}$. Par ailleurs, rien ne permet d'établir une filiation directe entre cette tradition et la ville de Pergame. Car s'il est vrai que l'unique représentation grandeur nature de ce dieu s'y trouvait, et probablement aux côtés d'une statue de Magna Mater de qualité équivalente, ces statues ne disent rien à propos d'une véritable vénération d'Attis à 
Pergame, où pas une figurine de ce dieu attribuée avec certitude n'a été conservée ${ }^{28}$. La connaissance du culte d'Attis était sans doute parvenue à Rome via les lieux de culte de Grèce et d'Italie du Sud, peut-être aussi via la place d'Attis dans le sanctuaire pergaménien.

La diffusion sur le Palatin des figurines d'Attis avec la pomme de pin en tant que matériel votif (fig. 10 a) peut contribuer à expliquer la popularité de ce dieu. Magna Mater et Attis sont liés dans une version du mythe au mont Ida, près de Troie. Magna Mater est désignée par l'épithète correspondante d'Idaea qui - comme expliqué plus haut - acquiert à Rome une grande popularité. Plus tard, Attis et la pomme de pin sont associés tant dans les représentations que dans les textes, et les auteurs de l'époque impériale parlent de troncs de pin venant du mont Ida, avec lesquels le navire de Magna Mater aurait été fabriqué. Les terres cuites d'Attis peuvent ainsi être interprétées comme une allusion - présente uniquement dans le matériel archéologique de Rome, et cela, dès la période républicaine - à la tradition mythique qui associe le mont Ida, Troie et Énée comme lieu d'origine et ancêtre de Rome ${ }^{29}$. La ferveur avec laquelle les Romains vénéraient Attis à l'époque républicaine est en revanche difficilement explicable et doit sans doute être considérée comme une tradition cultuelle spécifique au lieu.

En résumé, le parcours de Magna Mater depuis l'Asie mineure vers Rome et l'Italie se présente comme suit: des spécialistes religieux revêtant le statut de prêtres, des objets de culte et autres signes religieux (symbole de la pierre et habit sacerdotal) sont transmis à Rome, au sein d'une population déjà familiarisée dans une certaine mesure avec le culte de la déesse par le biais de ses sanctuaires de Grèce et d'Italie du Sud, mais familiarisée aussi, et ce n'est pas le moindre, avec la personne du dieu Attis. Ce moment est le seul dans la transmission du culte où l' "élément étranger » joue de toute évidence un rôle. Les prêtres d'Asie mineure et leur connaissance du rituel représentent la partie "étrangère » et donc supra-régionale du culte, à laquelle une partie romaine vient d'emblée s'ajouter. Dans le lieu de culte et les fêtes cultuelles, ce sont les éléments romains qui dominent: la vénération de Magna Mater est transformée par la société réceptrice.

\section{De Rome aux villes de l'Empire romain}

\subsection{Nouvelles fêtes, nouveaux cultores et diffusion du culte dans les provinces}

La diffusion du culte de Magna Mater depuis Rome vers les villes de l'Empire fait suite à une transformation du paysage cultuel de la Rome antique due à l'introduction de quelques nouveautés qui doivent avoir également concerné les communautés urbaines extérieures à Rome. Leur processus de diffusion à partir de Rome offre une fois encore la possibilité de s'interroger sur les mécanismes de transmission et la nature des communicateurs. Voit-on se dessiner dans les villes de l'Empire romain des formes et des pratiques cultuelles locales, ou bien le culte de la ville de Rome et du sanctuaire palatin est-il déterminant? Si oui, comment et par quel intermédiaire cette transmission s'effectue-t-elle?

Si l'on examine rapidement les éléments de la pratique cultuelle propres à la période impériale, on ne tarde pas à comprendre pourquoi la vénération de Magna Mater a pu 
facilement s'implanter dans d'autres lieux. Les fêtes et les rituels se modifient : depuis l'époque augustéenne, une deuxième fête en l'honneur de Magna Mater s'est ajoutée aux Megalesia ; elle est célébrée en mars et liée au rite de la lavatio. À partir de là, on voit se développer dans le courant du $\mathrm{I}^{\mathrm{er}}$ siècle ap. J.-C. un cycle de fêtes dédiées au dieu Attis qui meurt puis renaît (dendroforia [ou arbor intrat], dies sanguinis, lavatio). Ces fêtes nécessitent l'intervention de personnel - comme les dendrofori ou différents musiciens $\mathrm{s}^{30}$. Sous Antonin le Pieux, les cannoforia (ou canna intrat) - dont les exécutants se rassemblent comme les dendrofori en collèges - sont probablement fixées en mars, au début du cycle des festivités, et le rite du taurobolium est intégré dans la vénération de Magna Mater. Le cercle des personnes impliquées dans le culte s'élargit de manière sensible. Grâce aux processions et aux cortèges, les festivités deviennent à la fois plus spatiales, plus perceptibles et plus visibles. C'est un fait évident quand on compare la fête de mars aux ludi Megalenses, strictement liées au lieu, et aux mutitationes, limitées au cercle des familles patriciennes.

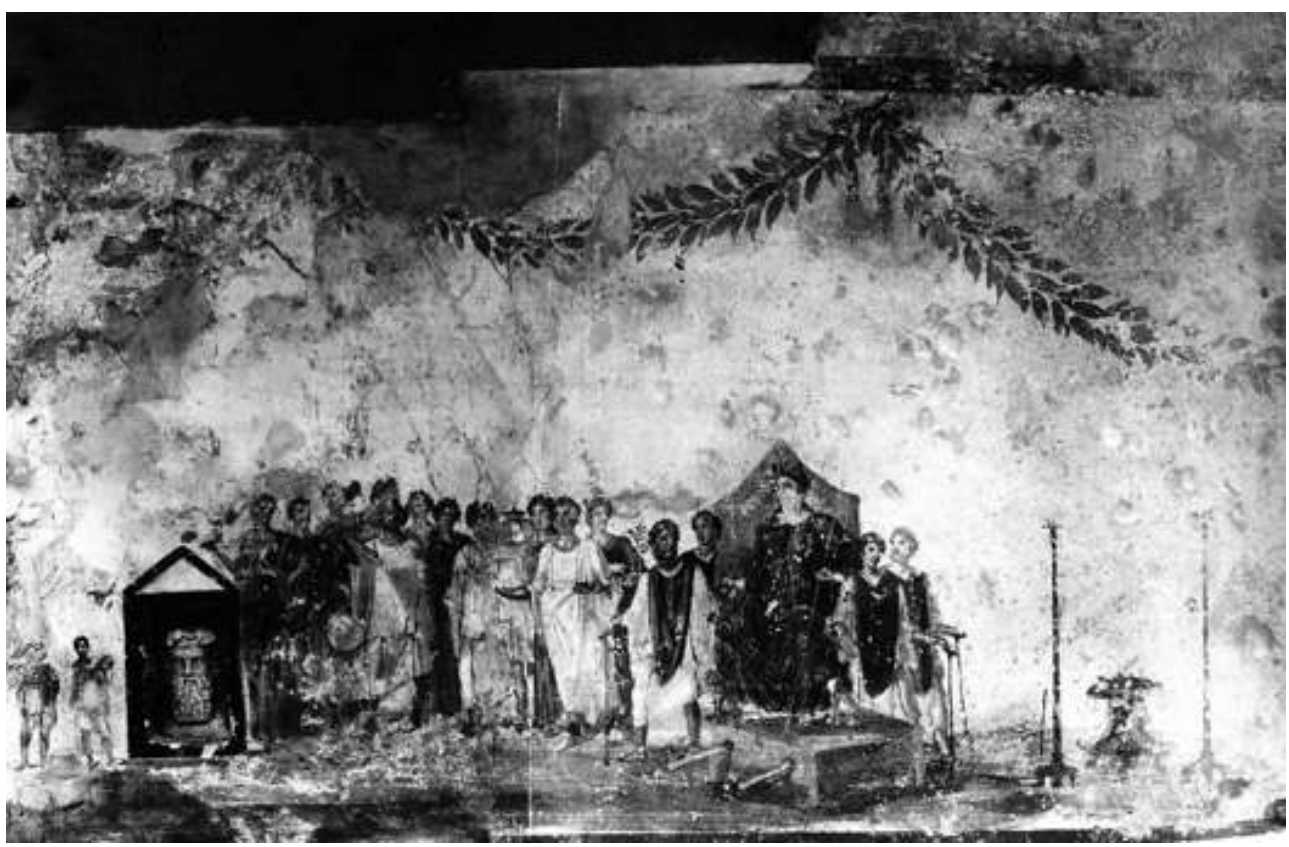

Fig. 11. Fresque de façade pompéienne avec représentation d'une procession en l'honneur de Magna Mater (Maison IX, 7, 1). Thomas Fröhlich, Lararien- und Fassadenbilder in der Vesuvstädten. Untersuchungen zur « volkstümlichen » pompejanischen Malerei, 1991 (MDAIR, supplément XXXII), pl. 59, 2.

Si les sources littéraires impériales soulignent la plupart du temps le caractère "phrygien » et «orgiaque » de ces événements, les représentations en donnent une tout autre image : on y voit des processions aux nombreux participants, qui jouent de la musique, font des libations ou assistent simplement à l'événement, et transportent les accessoires habituels de la sphère cultuelle romaine pour ce type de cortège, des accessoires sans lien avec les traditions spécifiques du pays d'origine de Magna Mater (fig. 11-12). À l'époque impériale aussi, on célèbre les fêtes de Magna Mater sous une forme romaine ${ }^{31}$. 


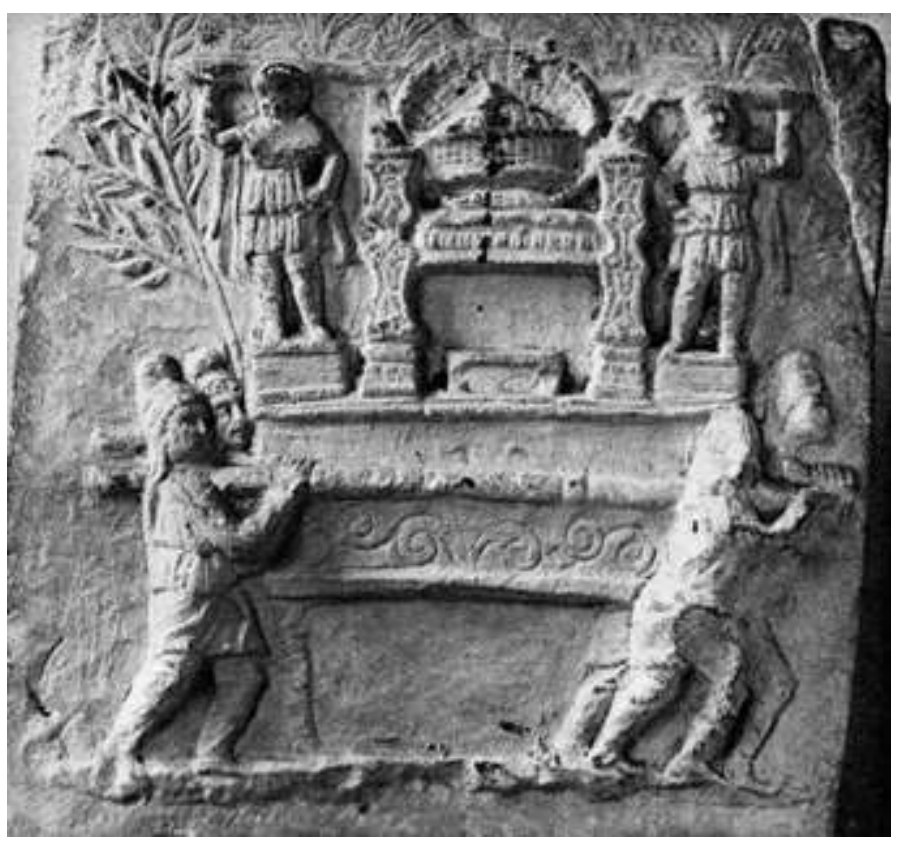

Fig. 12. Autel avec représentation d'un ferculum de Magna Mater et d'un pin décoré. Cambridge, Fitzwilliam Museum, CCCA, VII, 1977, n 39, pl. 28.

30 Ces nouvelles fêtes de mars dédiées à Magna Mater, nécessitant du personnel, sont transmises depuis l'Urbs aux villes de province. Nous le savons par le collège de dendrophores, attesté autour de la Méditerranée à partir du $\mathrm{I}^{\mathrm{er}}$ siècle ap. J.-C. et dont l'existence ne s'explique qu'en lien avec le culte de Magna Mater et le nouveau cycle de fêtes. Les cannoforia, célébrées depuis le II ${ }^{\mathrm{e}}$ siècle, ne semblent pas avoir attiré dans la même mesure des collèges indépendants - elles sont jusqu'ici attestées épigraphiquement dans six villes d'Italie ${ }^{32}$. Deux facteurs sont déterminants dans la transmission de ces fêtes cultuelles propres à l'Urbs. D'une part, elles sont pilotées par une institution de la ville, les quindecemviri sacris faciundis. On note que l'influence de cette assemblée religieuse de l'Urbs, à laquelle incombe le contrôle des cultes romains, s'étend également aux institutions extérieures à la ville. Dans les villes de province, les prêtres et collèges du culte de Magna Mater étaient subordonnés au quindecemviri. L'autonomie dont jouissaient les communautés dans les affaires religieuses n'était donc pas accordée dans le cas de Magna Mater; l'exécution de son culte et l'affectation des fonctions cultuelles étaient au contraire dirigées par un organisme central ${ }^{33}$. Cependant, l'adoption d'une nouvelle fête ne peut être entreprise que si des cercles de population sont disposés à la célébrer. L'élargissement mentionné plus haut du cercle des participants au culte de Magna Mater en constitue donc une condition importante. Tout comme des vicomagistri et des seviri augustales sont introduits pour honorer le récent culte impérial sous le principat, les changements de société de l'époque impériale ont pour conséquence d'ouvrir des fonctions publiques aux couches de liberti et autres personnes qui en étaient exclues auparavant et de leur permettre d'apparaître en public ${ }^{34}$. On peut donc concevoir le processus de reprise des fêtes de mars dans les villes comme la combinaison d'une influence accrue de Rome au niveau institutionnel et d'exigences de nature sociopolitique dans les populations.

Sur un plan dont on peut moins facilement identifier les acteurs, la diffusion des connaissances relatives au culte de Magna Mater est également perceptible dans ses dénominations. Les noms des lieux de son culte dans l'Urbs se retrouvent dans d'autres 
villes : le sanctuaire du Palatin autant que celui du Vatican acquièrent une notoriété suffisante pour que leur appellation soit reprise dans les villes de l'Empire. Le siège de la déesse sur le Palatin est pris comme modèle à Marseille, où Magna Mater reçoit l'épithète de Palatina. Le sanctuaire de l'ager Vaticanus est quant à lui copié notamment à Lyon et à Mayence ${ }^{35}$. Les sources ne disent pas dans quelle mesure les contenus sont transportés en même temps que les appellations, et si l'architecture et les fêtes sont également copiées. Comme ces reprises restent limitées à certaines villes, on peut supposer que la diffusion de dénominations romaines était non pas le résultat d'une décision centrale, mais celui de contacts personnels.

\subsection{Magna Mater à Ostie : espaces, images, collegia}

Si les preuves que les célébrations de mars en l'honneur de Magna Mater furent exportées dans les communautés de l'Empire existent dans les sources archéologiques, les transformations régionales qu'elles subirent sont nettement plus difficiles à cerner. Ostie représente une exception dans la mesure où son matériel offre une image différenciée, susceptible de révéler certaines conditions d'apparition de particularismes locaux. L'exemple de la ville portuaire permet de découvrir à la fois des spécificités locales du culte de Magna Mater et des rapports étroits avec Rome et la pratique cultuelle de cette dernière.

La phase la plus ancienne attestée archéologiquement du sanctuaire de Magna Mater à la Porta Laurentina remonte à l'époque augustéenne ${ }^{36}$. Le lieu se caractérise par la répartition délibérée de plusieurs bâtiments sur une aire étendue. Le temple de la mère des dieux, dans l'angle occidental de la place, occupe, en proportion des autres bâtiments et de la superficie totale, relativement peu de place. Le bâti se concentre surtout dans l'angle est, réservé au sanctuaire de Bellone et à l'Attideum (fig. 13). Il est placé derrière une vaste surface dégagée, dont on sait qu'elle était nommée dans l'Antiquité Campus Matris Deum ${ }^{37}$. La présence d'un espace consacré aux activités cultuelles, aux fêtes et aux jeux semble être un élément fondamental commun à Rome et à Ostie. Pour le reste, la conception spatiale de l'aire d'Ostie et celle du sanctuaire du Palatin sont assez différentes. 


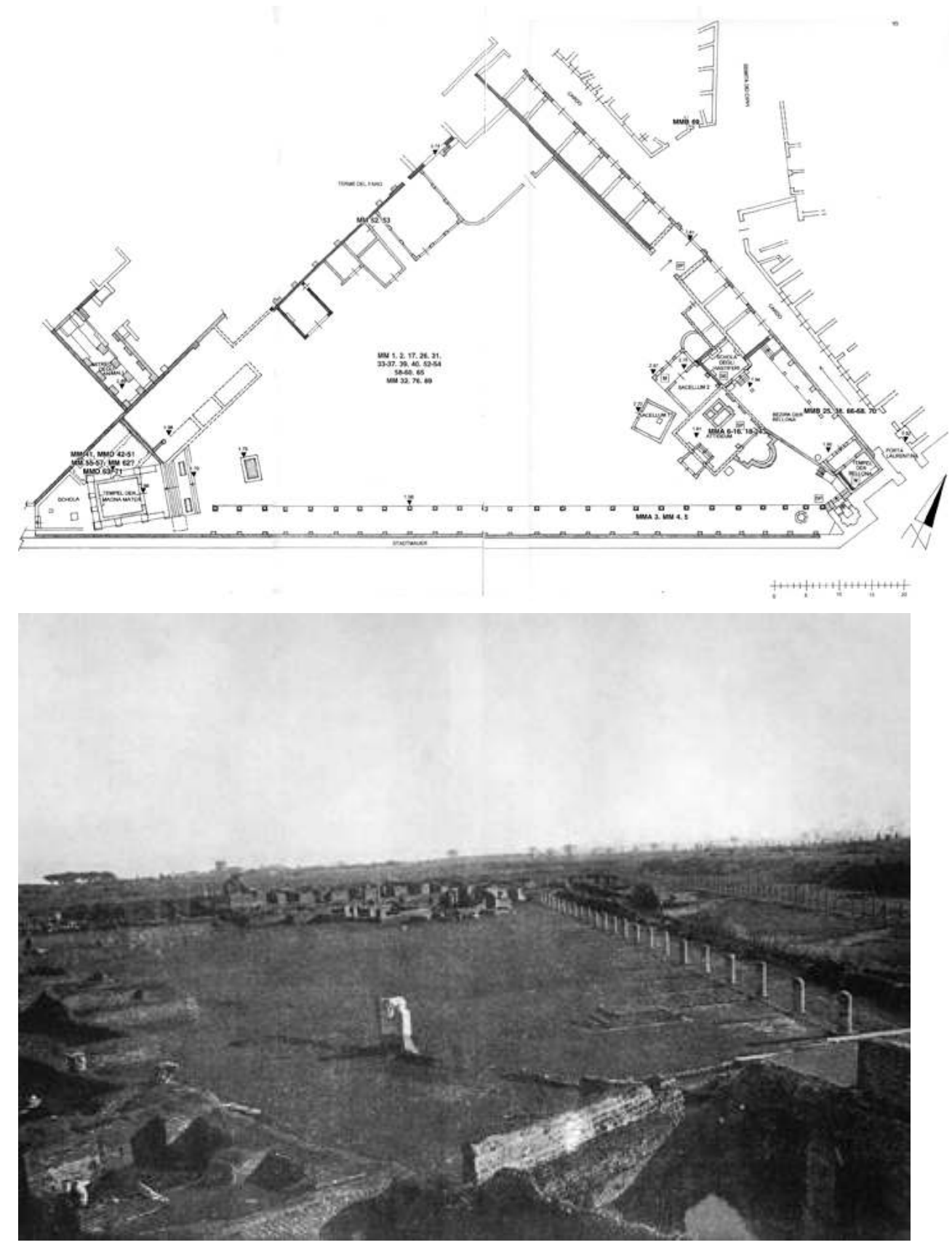

Fig. 13 a. b. Plan et vue (depuis l'ouest) du sanctuaire de Magna Mater à Ostie, Rieger (2004), pl. 61, 62.

La forme du sanctuaire et les témoignages de l'activité cultuelle liée à Magna Mater dans l'Ostie impériale révèlent une adaptation de type sociopolitique de sa vénération. Le fait de disposer d'espace fait du cas d'Ostie, florissante cité impériale, une exception et représente une réelle valeur économique. Cette spécificité du sanctuaire témoigne des moyens financiers des personnes engagées ici, dont l'appartenance à la classe sénatoriale est attestée par des inscriptions ${ }^{38}$. D'après celles-ci, la plupart des utilisateurs de l'aire sont membres des collegia, qui sont dans le sanctuaire d'Ostie particulièrement présents, comparés à ce qui se passe dans d'autres villes. La situation économique et sociale particulière de la cité portuaire favorise en général la vie associative, qui intervient dans tous les domaines de la vie. Vu la position de la cité, important centre de commerce, et les conditions de vie qui y sont liées, la nécessité de 
s'organiser en collèges semble en effet y avoir été plus aiguë qu'ailleurs : résumé assez grossièrement, les associations garantissaient la défense des intérêts, l'intégration et le sentiment d'appartenance. Il n'est donc pas étonnant d'y voir des dendrofori et des cannofori s'engager plus résolument que dans d'autres villes, ou des associations particulières, comme les hastiferi - porteurs de lances, attestés ici depuis le II ${ }^{\mathrm{e}}$ siècle ap. J.-C. et en dehors d'Ostie à trois endroits seulement - se former dans le cadre de la vénération de la déesse ${ }^{39}$. On peut donc affirmer qu'à partir de la constellation économique et sociale locale et en dépit du lien étroit entretenu avec Rome tant sur le plan architectural que sur le plan social, une vénération spécifique de Magna Mater qu'on ne trouve sous cette forme nulle part ailleurs - se développe à l'embouchure du Tibre.

Même si, étonnamment, aucune trace d'une vénération de Magna Mater n'a été trouvée à Ostie à l'époque républicaine - alors que le navire portant la pierre noire y est passé en remontant le Tibre - on trouve plus tard des représentations de l'arrivée de la déesse dans la statuaire, dont la forme et le vocabulaire trahissent d'autres aspects des liens supra-régionaux et des particularités locales du culte que ce que l'on trouve dans les textes. Un motif précis de Magna Mater fréquent à Rome comme à Ostie, présent sur un medium peu visible, ne s'est diffusé que dans les deux villes fluviales et s'explique à la lumière de l'histoire locale. Des antéfixes dont les matrices sont utilisées depuis l'époque augustéenne jusqu'au II siècle ap. J.-C. montrent en effet Magna Mater trônant sur un navire - il s'agit donc d'une représentation de son arrivée en terre italique, qui, de fait, est liée à la légende évoquant l'échouement de l'embarcation et son sauvetage miraculeux par la vestale Claudia (fig. 14) ${ }^{40}$.
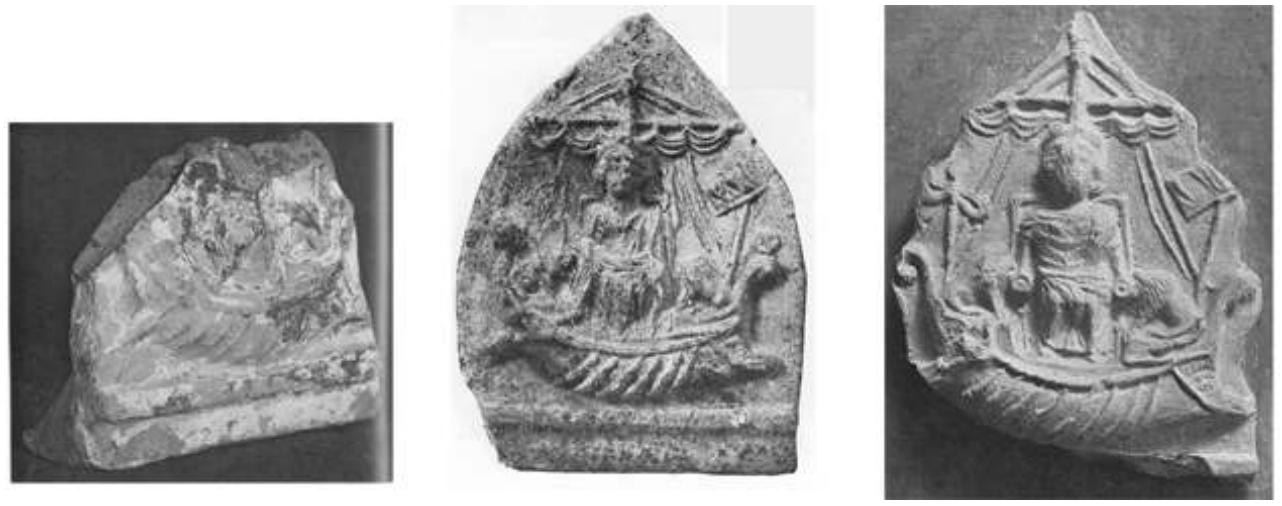

Fig. 14. Antefixes de terre cuite avec représentation de Magna Mater provenant du Palatin et d'Ostie. Rome, Musée du Palatin et Ostie, Antiquarium. Fragment Palatin : CCCA, III, 1977, n 11, pl. 20 ; Piazzale delle Corporazioni. ibid., n 427, pl. 269 ; sanctuaire de Magna Mater, ibid., n³ 397, pl. 247.

$\mathrm{Vu}$ la position qu'elle occupe sur le côté de la gouttière, une antéfixe ne constitue pas un vecteur iconographique très efficace. Aussi, les fragments trouvés n'appartiennent pas par hasard à des édifices plus petits, proches du temple de Magna Mater sur le Palatin, au Campus de Magna Mater à Ostie ainsi qu'à un autre édifice d'Ostie, le Piazzale delle Corporazioni ${ }^{41}$. Ces représentations évoquent l'événement bien connu de l'arrivée de la mère des dieux, véhiculant ainsi cette histoire importante pour Rome et pour Ostie. Les événements politiques et les contenus religieux trouvent ainsi une diffusion limitée via les matrices de sculpture. Ces antéfixes possèdent de par leur motif une valeur spécifiquement locale, distincte de celle des représentations de la mère des dieux trônant, que l'on rencontre habituellement sur les fonds de lampes à l'huile, ou de l'Attis trapézophore, deux types de représentations faisant partie du répertoire 
iconographique courant de l'Empire et qui, de manière significative, sont presque absents à $\mathrm{Ostie}^{42}$. Les antéfixes sont peut-être peu visibles, mais elles mettent en exergue une particularité locale de la vénération de Magna Mater, qui ne revêt pas d'importance dans d'autres villes.

La proximité géographique et les rapports étroits existant entre Rome et Ostie permettent à eux seuls de supposer que beaucoup de cultores connaissaient à la fois le sanctuaire du Palatin et celui d'Ostie. Cette hypothèse est confirmée par des indices dans le matériel archéologique. La présence d'un type précis de Vénus à la fois sur le Palatin et à Ostie montre en effet qu'un échange s'est produit au sein de cercles d'adorateurs qui n'avaient pas de fonction particulière dans le culte. Dans un ensemble d'ex-votos du sanctuaire d'Ostie datées du II siècle ap. J.-C. on trouve trois statuettes de Vénus assez insignifiantes, du type Louvre-Naples (fig. 15).

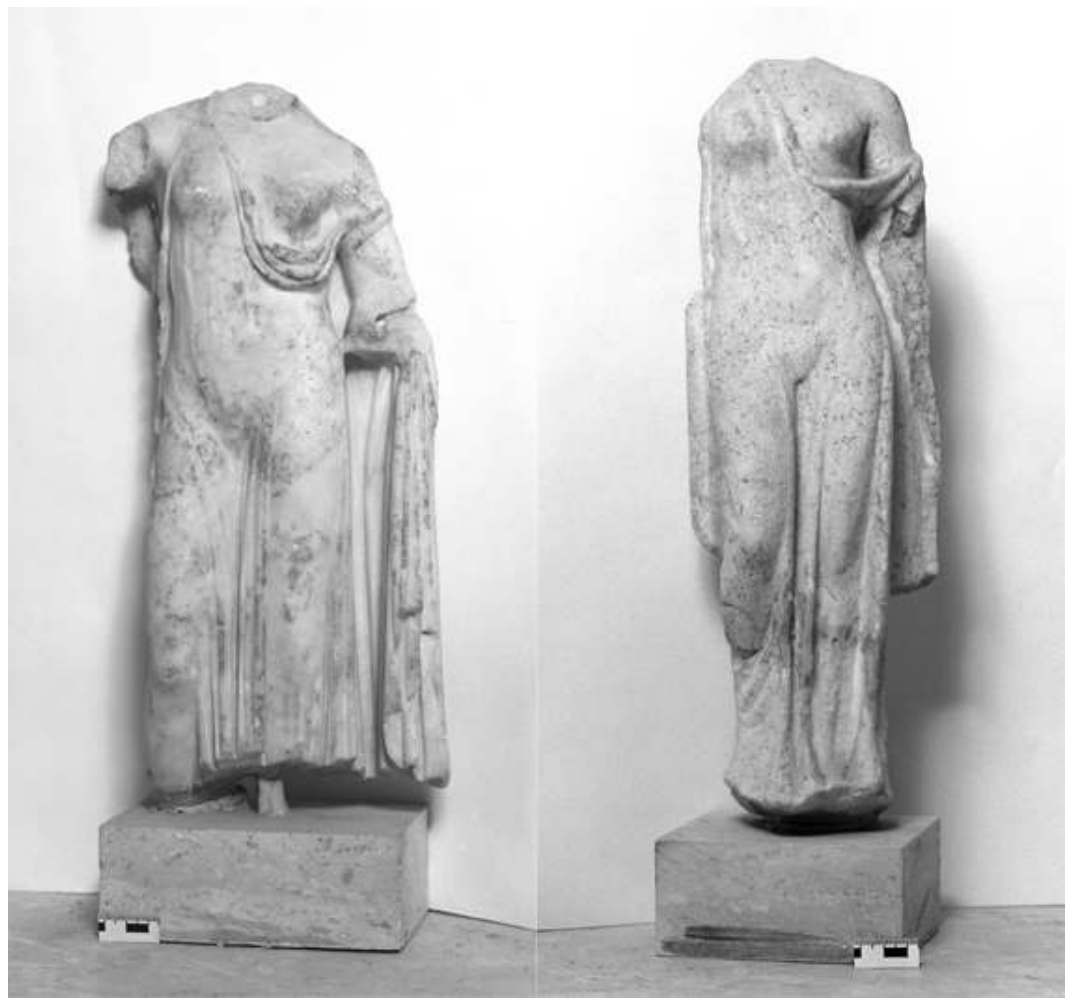

Fig. 15. Vénus de l'Attideum du sanctuaire de Magna Mater d'Ostie, Antiquarium („e siècle ap. J. C.) Rieger (2004), fig. 102 b., C.

Une Vénus du même type, mais démesurée par rapport au lieu, se trouvait depuis le $\mathrm{I}^{\mathrm{er}}$ siècle ap. J.-C. dans le sanctuaire de Magna Mater au Palatin (fig. 16) ${ }^{43}$. 


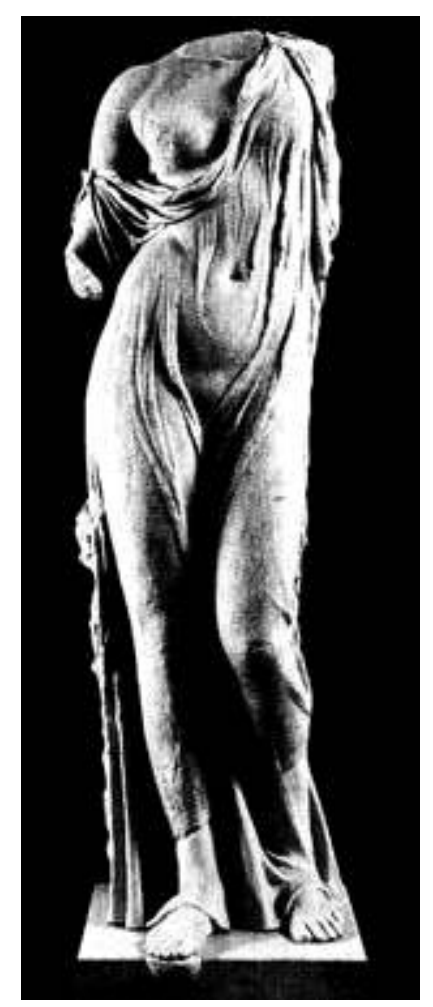

Fig. 16. Vénus du sanctuaire de Magna Mater sur le Palatin, Musée du Palatin (fin du ır siècle ap. J. C.). Tomei( 1997), p. 127, cat. 105.

Ce type, qui se caractérise par un chiton fluide, menaçant de glisser de l'épaule, a été utilisé pour représenter la Vénus Genetrix, ancêtre féminin de la dynastie julioclaudienne. Comme les Julio-Claudiens se réclamaient d'Énée, héros troyen déjà évoqué à plusieurs reprises dont la mère était Vénus, la sculpture de la Vénus Genetrix de Rome est porteuse d'un message politique clair : elle est à travers la lignée du roi d'Alba Longa et de son fondateur Énée la mère de la première maison impériale romaine ${ }^{44}$. Depuis le début de l'Empire, ces Vénus sont des figures populaires, que l'on place notamment dans les thermes, mais que l'on offre aussi comme ex-votos. Leur accumulation dans le sanctuaire d'Ostie semble toutefois indiquer que le fondateur du lieu, Cartilius Euplus, voulait imiter le sanctuaire palatin, qu'en tant qu'habitant d'Ostie il avait pu voir de ses propres yeux. Le lien politico-religieux entre Vénus et Magna Mater, spécifique à Rome, se prolonge ainsi à Ostie.

\subsection{Contacts personnels outre-mer}

Après avoir envisagé sur la base des exemples de Rome et d'Ostie le domaine des utilisateurs «normaux », il convient maintenant d'examiner les sphères d'action et les contacts des spécialistes religieux.

Une inscription trouvée dans une taverne installée sous les escaliers du temple palatin fait référence à un Caius Fonteius Doryphorus, sacerdos M(atris) et Atti(di)s à l'époque antonine ; un autre témoignage qui le nomme avec cette même titulature a été trouvé dans le nord du Palatin. Bien loin de là, à Carthage, un sacerdos M(atris) D(eum) M(agnae) I(deae) de même nom apparaît avec une dédicace à Asclépios ${ }^{45}$. La pierre portant l'inscription, également à dater de l'époque antonine, a été découverte sur le versant oriental de la colline de Byrsa, en dehors de son contexte d'origine. On peut toutefois 
supposer qu'elle provient du sanctuaire d'Asclépios qui se trouvait sur la même colline ; le temple de Magna Mater ne se trouvait pas loin de là (fig. 17) ${ }^{46}$.

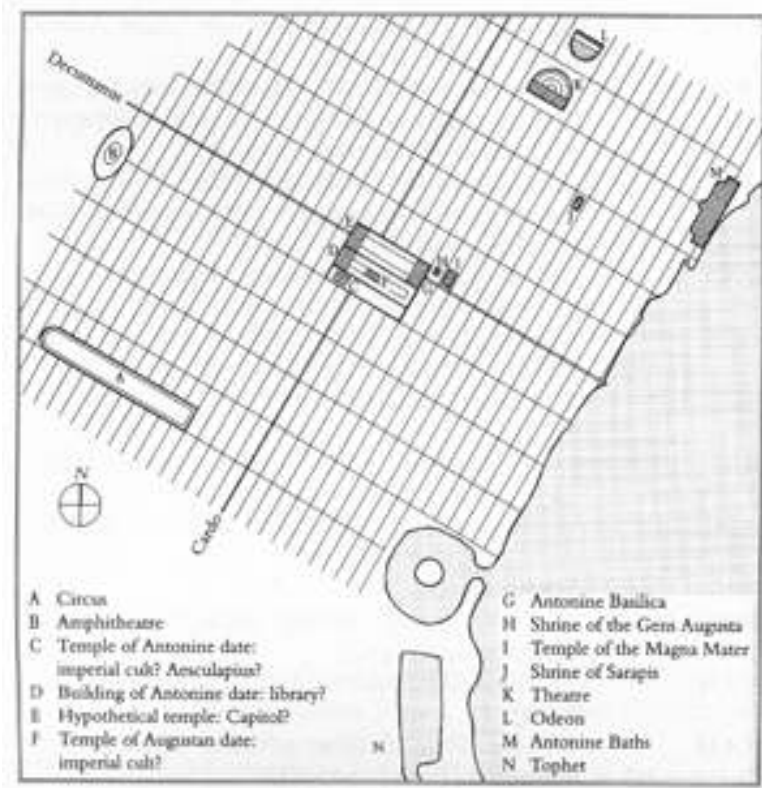

Map r: Plan of Carthage in the Antonine Period

Fig. 17. Plan de la colline de Byrsa, à Carthage, avec les sanctuaires qui s'y trouvent. James B. Rives, Religion and Authority in Roman Carthago from Augustus to Augustine, Oxford, Clarendon Press, 1995, carte 1.

Si l'on admet que le Fonteius romain est identique à celui de Carthage, un prêtre a quitté Rome pour Carthage au $\mathrm{II}^{\mathrm{e}}$ siècle ap. J.-C. - notons que des relations commerciales directes sont attestées par une statio des marchands carthaginois sur le Piazzale delle Corporazioni d'Ostie ${ }^{47}$. S'il était actif ici dans les intérêts de Magna Mater, et de quelle façon, nous ne pouvons pas le dire, mais sa consécration à Asclépios prouve quoi qu'il en soit qu'il a accompli des actes religieux dans son lieu de visite. Rien n'indique pour ce Fonteius une origine d'Asie mineure, ce qui permettrait de le considérer d'une certaine façon comme vecteur particulier du savoir lié au culte de Magna Mater. Mais un homme chargé comme lui d'une fonction de sacerdos emmenait d'office avec lui le savoir, la connaissance et les particularités du culte local, d'autant que, comme on le sait, certains changements cultuels interviennent à Rome à l'époque antonine. Fonteius, qui était actif à Rome, aurait très bien pu faire rapport du déroulement des nouvelles festivités ${ }^{48}$. Si nous ignorons dans quelle mesure de telles connaissances ont pu être transmises lors d'un échange avec un autre prêtre, nous possédons du moins des preuves que les prêtres disposaient d'un large rayon d'action, qui doit avoir abouti à des échanges et à des contacts personnels, ne serait-ce que parce que Fonteius fut reçu en hôte.

Les relations mises au jour entre les différents sanctuaires de Magna Mater, qui peuvent avoir existé en termes de conceptions de l'espace, de statuaire, de noms et d'expérience individuelle des cultores et spécialistes religieux, peuvent donner une idée de la manière dont, malgré la présence d'éléments supra-régionaux telles les fêtes de mars, de nombreuses traditions locales eurent l'occasion de se développer. 


\section{Flash-back sur l'Asie mineure : images et édifices}

\subsection{Cyzique}

41 Pour terminer, tournons-nous à nouveau vers l'Asie mineure - région à partir de laquelle la déesse a été introduite et dont les sanctuaires dédiés à son culte n'ont cessé de rayonner vers l'Occident. Le processus des reprises, en effet, se poursuit sans interruption. Ainsi, Ostie emprunte à Cyzique, sur la mer de Marmara, des éléments d'une construction qu'il faut probablement identifier au Métroon de la ville, connu par ailleurs. On y trouvait de nombreuses caryatides, sculptées à l'effigie de Pan et réalisées à la fin du $\mathrm{II}^{\mathrm{e}}$ siècle ap. J.-C. (fig. 18) ${ }^{49}$.
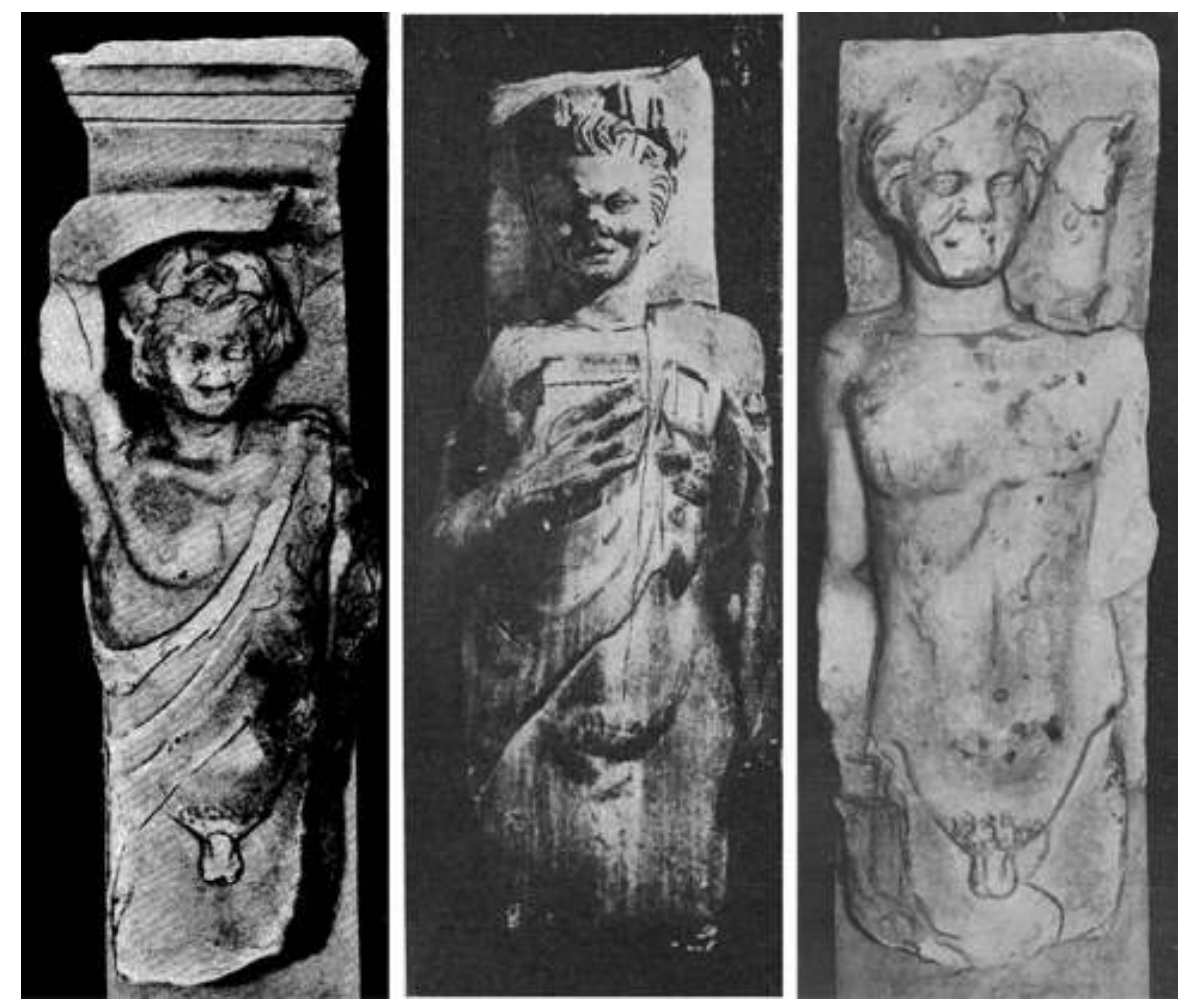

Fig. 18. Figures de Pan provenant d'un Métroon ( ?) de Cyzique. Istanbul, Musée archéologique. Devambez, 1937, p. 178-194, fig. 1, 3 et 6.

Elles représentent le dieu jeune et imberbe, vêtu d'une nebris ou nu. Une ressemblance frappante existe entre les pièces de Cyzique et les figures adossées aux colonnes de l'Attideum d'Ostie, qui doivent être situées dans la première moitié du III ${ }^{\mathrm{e}}$ siècle ap. J.-C. (fig. 19) $)^{50}$. 

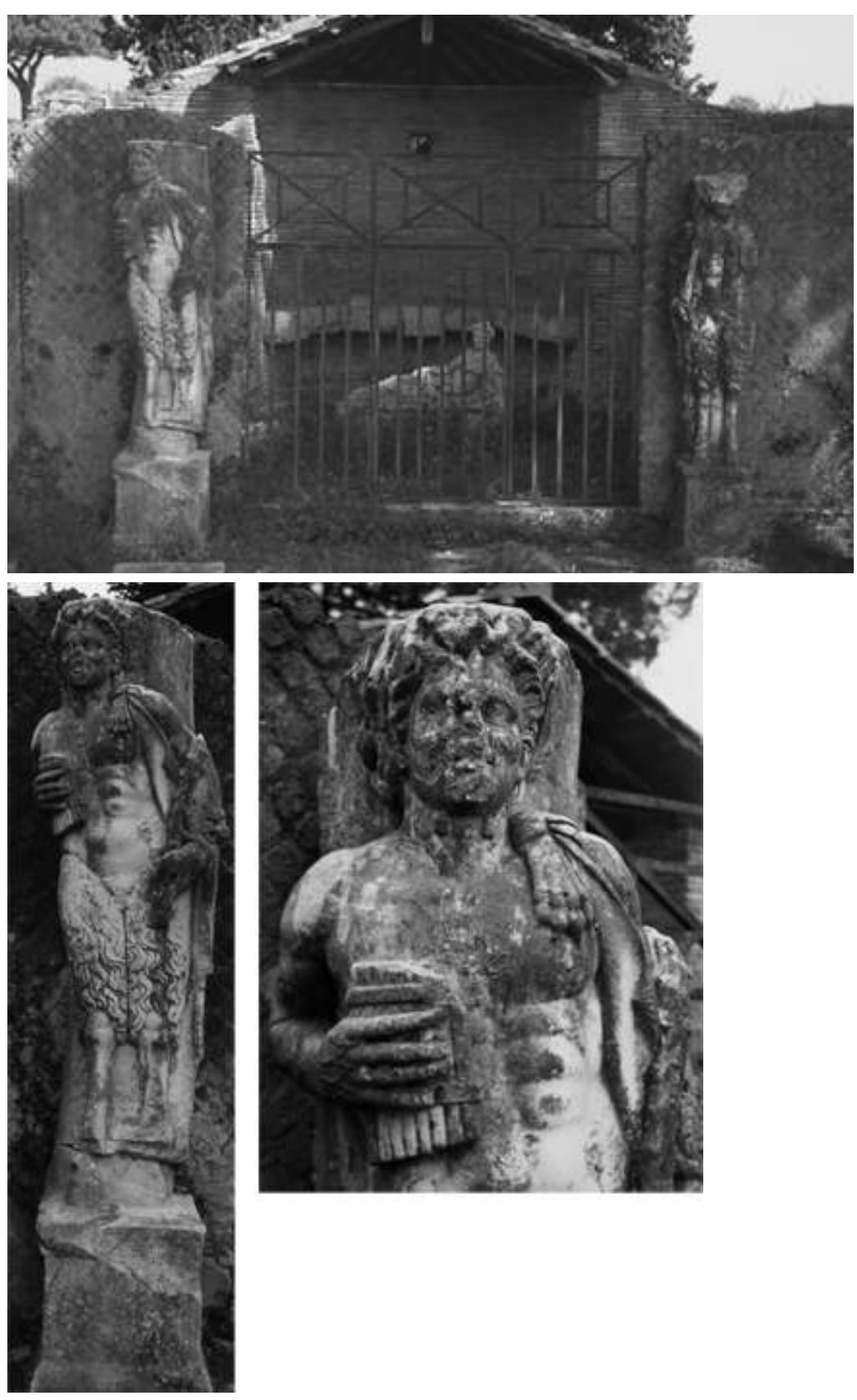

Fig. 19 a. b. Attideum du sanctuaire de Magna Mater d'Ostie et détail d'une figure de Pan. Photos de l'auteur.

Si le motif du jeune Pan appliqué à un pilier de soutènement n'est connu que par ces deux exemples, celui du Pan barbu et plus âgé est en revanche habituel. Les ressemblances observées vont jusqu'à certains détails de la chevelure et du corps, si bien que plutôt que de voir là une simple concordance iconographique, on peut imaginer que le modèle des pièces de Cyzique a dû être connu du sculpteur du Pan d'Ostie. Non seulement ces figures du sanctuaire d'Ostie semblent avoir été influencées par la ville d'Asie mineure, mais le grand Attis couché de l'Attideum d'Ostie trouve également son modèle dans une statue de Cyzique : au vu de ces éléments, l'hypothèse de contacts entre les deux villes est tout à fait plausible. L'Attis couché renvoie en effet par son attitude et son motif à une statue représentée sur les monnaies de Cyzique, qui correspond probablement à l'image cultuelle locale du dieu. Les relations personnelles, 
les contacts commerciaux et la signification du sanctuaire de Cyzique ont dû porter à la connaissance des habitants d'Ostie cette iconographie et susciter l'imitation, et pas seulement celle des caryatides ${ }^{51}$. Or, si l'Asie mineure continue à influencer l'Occident, Rome rayonne également en direction de celle-ci à l'époque impériale.

\subsection{Pessinonte}

Cette influence «à rebours » est perceptible à Pessinonte, là même où se trouvait autrefois la pierre de la mère des dieux. On assiste sous l'Empire à un transfert de formes architecturales qui plaide également en faveur de la reprise du rituel : certains détails du sanctuaire du Palatin semblent avoir été imités dans un temple exhumé à Pessinonte, actuelle Ballihisar. Ce temple jadis périptère se trouve dans le sud de la ville, sur la rive est du Gallos (fig. 20-22) ${ }^{52}$.

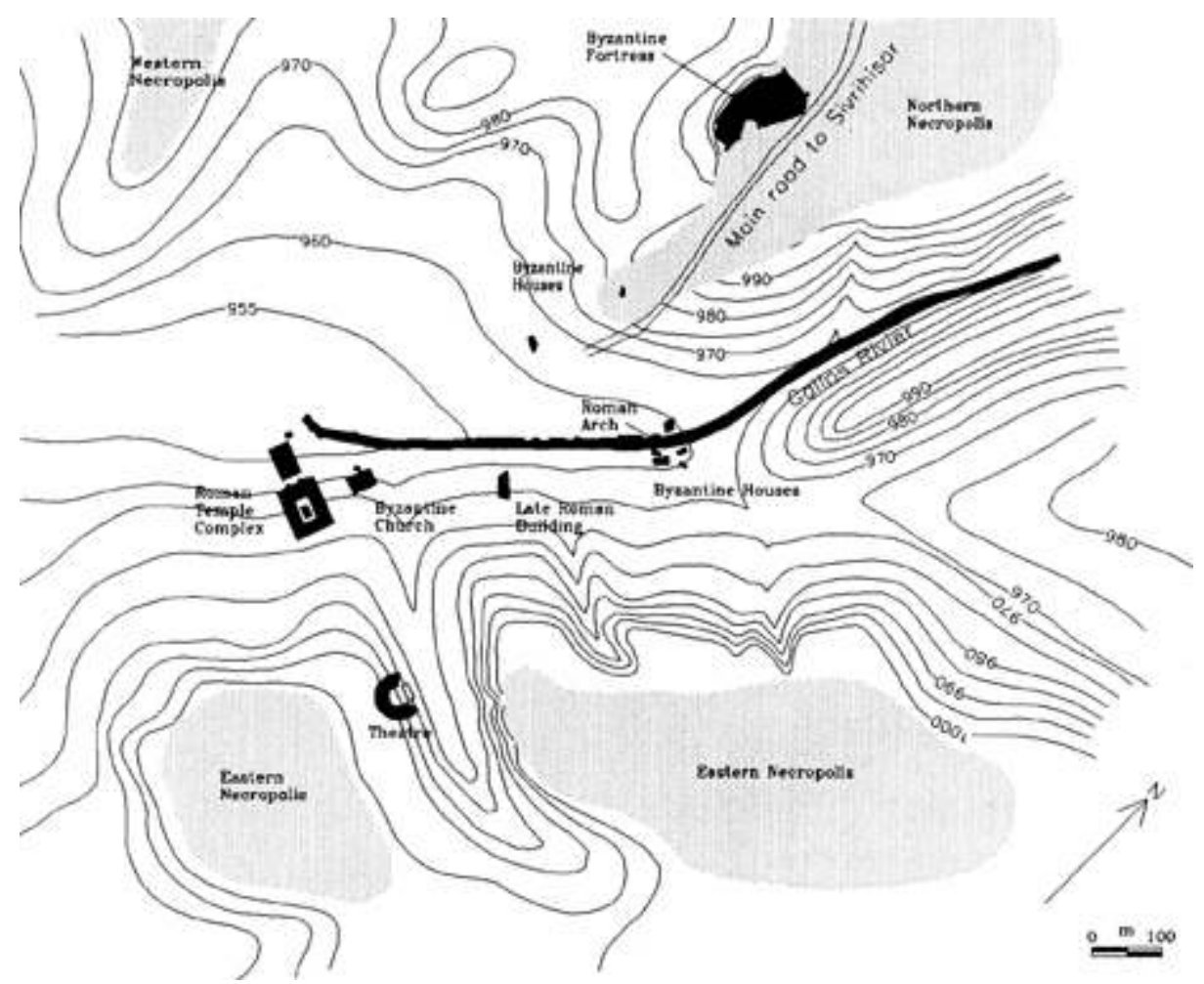

Fig. 20. Plan de Pessinonte avec le temple mentionné («Roman Temple Complex ». John Devreker Hugo Thoen, Frank Vermeulen, Excavations in Pessinus: The so-called Acropolis. From Hellenistic and Roman Cementery to Byzantine Castle, Gand, Academia Press, 2003 (" Archaeological Reports Ghent University », 1), fig. 4. 


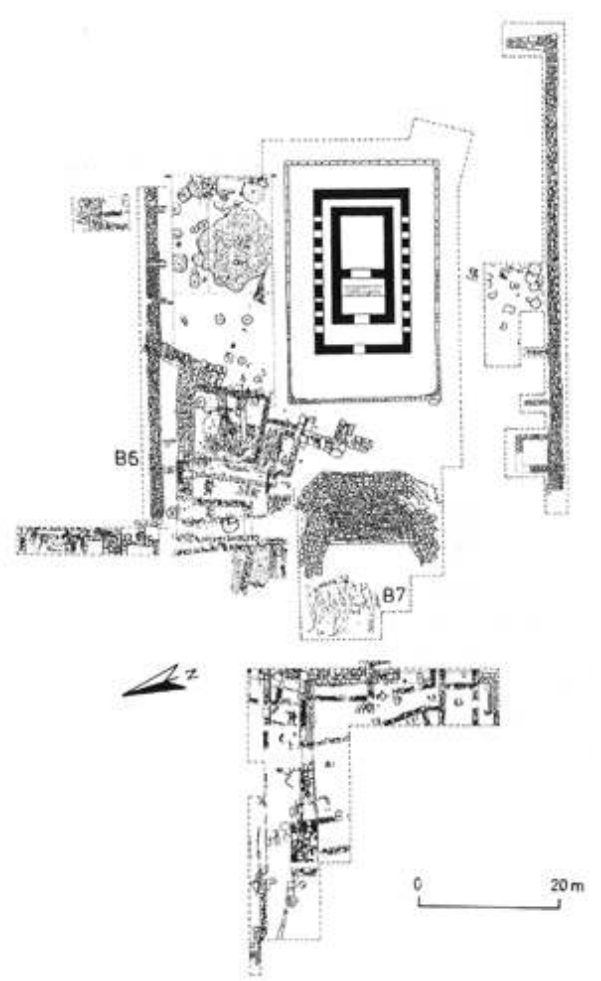

Fig. 21. Plan du temple de Pessinonte. Devreker / Waelkens (1984), fig. 2. inscriptions ni œuvres sculptées n'ont été trouvées, mais les archéologues parlent généralement d'un temple dédié au culte impérial. Leur argumentation se base en premier lieu sur l'époque de construction, à savoir le règne de Tibère, sous lequel le culte impérial fut introduit à Pessinonte, ainsi que sur la découverte d'inscriptions à « caractère officiel ». Cette identification à un temple dédié aux Divi est par ailleurs à juste titre mise en doute, d'autant plus que l'on a trouvé sous les constructions impériales des bâtiments antérieurs d'époque classique et hellénistique qui, si l'on se base sur leur caractère sacral, ne s'expliquent naturellement pas par ce culte impérial ${ }^{53}$. Trois observations font pencher la balance en faveur d'une association du temple d'époque impériale à la vénération de Magna Mater, dans laquelle celle des Divi est justement incluse de manière symbiotique. Comme dans d'autres villes de l'Empire, le culte impérial et celui de Magna Mater sont étroitement liés à Pessinonte ${ }^{54}$. En témoignent les inscriptions, qui nomment une même personne comme prêtre de l'empereur et de Cybèle. Ces fonctions religieuses sont ici réunies en une. La situation du temple, qui domine le fleuve Gallos, renvoie également à la déesse. C'est en effet sur ses rives que se déroule pour une part le mythe de Magna Mater, c'est également dans ce fleuve que la statue de culte est lavée à Pessinonte ${ }^{55}$.

Un autre critère important réside dans l'architecture même du temple. Celui-ci possède du côté fleuve un escalier aménagé en forme de tribune, petit théâtre qui a pu servir aux représentations rituelles telles que nous les connaissons par l'architecture et les sources écrites du temple du Palatin à Rome (fig. 22) ${ }^{56}$. 

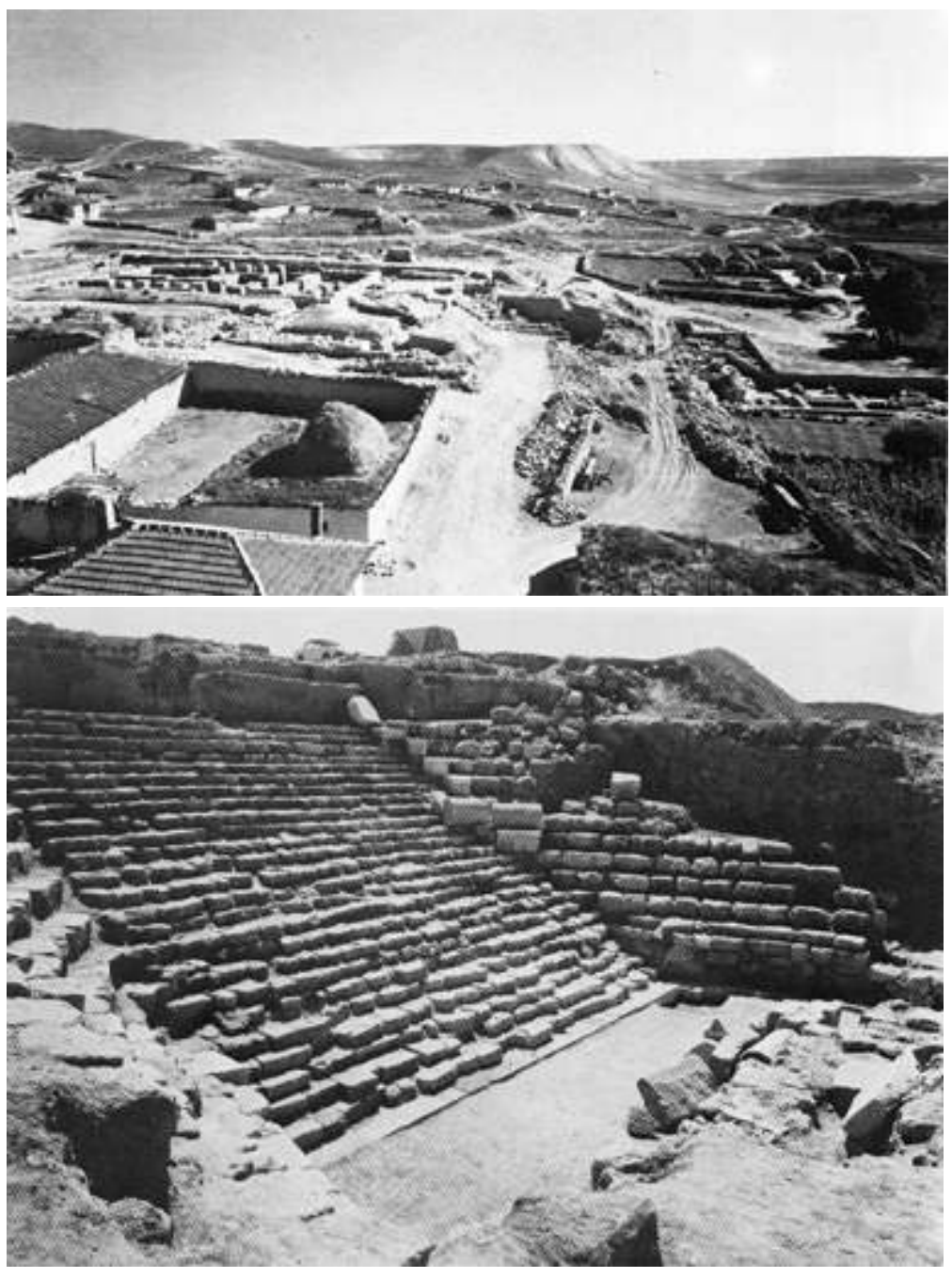

Fig. 22 a. b. Vue sur les fouilles du temple en direction du sud-ouest et sur les escaliers en forme de tribune précédant le temple. Devreker / Waelkens (1984), p. 20 sq., fig. 32, 34.

La portée des comparaisons architecturales est très faible - l'exemple du théatretemple montre précisément qu'elles peuvent induire des associations arbitraires entre bâtiments vaguement ressemblants et mener ainsi à des erreurs. En ce qui concerne le lien entre le temple et le théâtre, on peut néanmoins affirmer avec certitude qu'il s'agit d'une forme architecturale courante en Occident, alors que seul l'exemple de Pessinonte est connu en Asie mineure ${ }^{57}$. Il faut également considérer dans le débat concernant ces édifices, que tant à Rome qu'à Pessinonte, un terrain en pente a été utilisé pour la conception d'un espace dédié aux spectateurs et étroitement lié au temple. L'édifice cultuel et la tribune ainsi que les processions et les jeux qui en découlent sont apparemment communs aux deux lieux. Ce parallèle entre le berceau de Magna Mater et son temple à Rome ne me semble pas relever du hasard. On peut considérer cette forme architecturale comme une preuve du transfert dans l'autre sens de certains éléments cérémoniels, comme les représentations données devant le temple de Magna Mater. Le culte romain transformé de la déesse est ainsi réimporté dans sa région d'origine. 


\section{Magna Mater : une image inchangée dans un culte aux multiples facettes}

45 J'ai présenté quelques disiecta membra du culte de Magna Mater relevant de domaines très divers de la culture matérielle et immatérielle, parmi lesquels les acteurs, les édifices, les fêtes, les institutions religieuses, les images, les dénominations. J'ai tenté de montrer - sous l'angle de l'archéologie - les voies, les moyens et l'identité des vecteurs du culte de Magna Mater entre Asie mineure, Rome et les provinces et d'illustrer par là la complexité des modèles de diffusion, des fondements propres à la reprise partielle des signes religieux. À aucun moment, il ne s'agit d'importer le culte de Magna Mater tel quel. L'adaptabilité et la naissance de traditions cultuelles locales sont déterminées par l'adoption de la déesse dans des villes géographiquement très dispersées, aux situations culturelles et sociales très diverses - que l'on songe seulement aux très nombreuses formes préromaines de divinités maternelles rencontrées par Magna Mater en Gaule, en Afrique du Nord ou dans le Levant. Seule la statue hellénisée de la Magna Mater trônant, la tête ceinte d'une couronne murale (fig. 2), apparaît partout et, comparée à d'autres formes divines, sous une forme assez univoque. Elle apparaît donc comme une constante.

Ce sont tantôt des spécialistes religieux, tantôt des hommes sans fonction cultuelle particulière qui tiennent lien d'entremetteurs. Autant que nous sachions, des «Phrygiens » véritables, transportant avec eux symboles matériels (pierre de culte) et pratiques religieuses (accessoires, habit), n'apparaissent dans ce rôle qu'à Rome et dans la phase précoce du culte. De nouveaux éléments locaux - avant tout des festivités sont développés dans l'Urbs conformément à la tradition romaine. Le temple que l'on y érige correspond également aux conceptions religieuses de la société réceptrice.

Des variantes locales se laissent également identifier à plusieurs occasions. Dans ce cas, la connaissance que l'on y a des signes et pratiques religieuses propres à la vénération de Magna Mater influence directement l'avatar local de son culte. À l'époque républicaine, peu après l'introduction de la déesse à Rome, le héros Attis et la pratique votive qui lui est liée au Palatin servent semble-t-il d'exemple. La dispersion de certains motifs iconographiques a également montré qu'il existait une communication entre les sanctuaires et leurs utilisateurs respectifs à l'époque impériale.

La Vénus Genetrix ou la Magna Mater trônant sur un navire ont été utilisées comme thèmes à Rome et à Ostie, où leur message politique était le plus évident. Une diffusion supra-régionale ne se vérifie que dans le cas des fêtes instaurées sous l'Empire en mars et des collegia qui y sont liés. Cette expansion est contrôlée et favorisée par l'organe des quindecemviri, une institution de l'Urbs.

En résumé, on peut affirmer que de nombreuses variantes régionales sont observables : les éléments de culte supra-régionaux sont en revanche plutôt rares. Ainsi que la confrontation de la statuaire et de l'architecture en Italie et en Asie mineure l'a montré, la reprise des pratiques religieuses reste sélective, de même que les transferts et échanges sont un processus dynamique. Car la religion est une chose comme on le sait variable et adaptable, aussi protéiforme que les groupes qui la pratiquent. 


\section{BIBLIOGRAPHIE}

N. B. : Les auteurs de l'Antiquité sont cités d'après : Cancik, H., Schneider, H. (éd.) : Der Neue Pauly. Enzyklopädie der Antike, Stuttgart / Weimar, Verlag J. B. Metzler, 1996, t. 1, p. XXXIX-XLVII.

Bartolli, A. (1947) : Il culto della Mater Deum Magna Idaea e di Venere genitrice sul Palatino, Rome, Tipografia Poliglotta Vaticana.

Beard, M. / North, J. / Price, S. (1998) : Religions of Rome, vol. 1, Cambridge, Cambridge University Press.

Becatti, G. (1961) : Mosaici e pavimenti marmorei, Rome, Istituto Poligrafico dello Stato (Scavi di Ostia, IV).

Borgeaud, Ph. (1996) : La Mère des dieux. De Cybèle à la Vierge Marie, Paris, Seuil.

Calza, G. (1947) : « Il santuario della Magna Mater a Ostia », in : Memorie della Pontificia Accademia Romana di Archeologia, VI, p. 183-205.

CCCA : Corpus cultus Cybelae Attidisque, 7 vol., Vermaseren, M. J. (éd.), Leyde / New York, Brill, (EPRO), 1977 sqq.

Cecamore, C. (2002) : Palatium. Topografia storica del Palatino tra III sec. a. c. e I sec. d.c., Rome, L'Erma di Bretschneider (Bolletino della Commissione Archeologica Comunale di Roma, suppl. IX).

CIL : Corpus Inscriptionum Latinarum, 1863 sqq.

Coarelli, F. (1987) : I santuari di Lazio, Rome, Nuova Italia scientifica.

Coarelli, F. (1988) : Il Foro Boario. Dalle origine alla fine delle Repubblica, Rome, Quasar.

Cumont, F. (1906) : Les Religions orientales dans le paganisme romain, Paris, Librairie Orientaliste P. Geuthner. Nouvelle édition (2006) : Bonnet, C., Van Haeperen, F. (éd.), avec la collaboration de Toune, B., Turin, Nino Aragno (Bibliotheca Cumontiana, Scripta Maiora, 1).

Devambez, P. (1937) : « Deux Piliers décorés trouvés à Cyzique », in : Revue Archéologique, IX, p. 176-194.

Devreker, J. / Waelkens, M. (1984) : Les Fouilles de la Rijksuniversiteit te Gent à Pessinonte. 1967-1973, hommage à Pieter Lambrechts, Bruges, De Tempel (Dissertationes Archaeologicae Gandenses, XXII).

Devreker, J. / Thoen, H. / Vermeulen, F. (1995) : « The Imperial Sanctuary of Pessinus and its Predecessors. A Revision », in : Anatolia Antiqua, Eski Anadolu, III, p. 125-144.

Fishwick, D. (1966) : « The cannophori and the March Festival of Magna Mater ", in : Transactions and Proceedings of the American Philological Association, XCVII, p. 193-202.

Fishwick, D. (1967) : « Hastiferi », in : Journal of Roman Studies, LVII, p. 142-160.

Garland, R. (1987) : The Piraeus from the fifth to the first century B.C., Londres, Gerald Duckworth.

Graillot, H. (1912) : Le Culte de Cybèle. Mère des Dieux à Rome et dans l'Empire romain, Paris,

Fontemoing et $\mathrm{Cie}$, (Bibliothèque des Écoles françaises d'Athènes et de Rome, CVII).

Gruen, E. S. (1990) : Studies in Greek Culture and Roman Policy, Leyde, Brill.

Hanson, J. A. (1959) : Roman Theater-Temples, Princeton, Princeton University Press (Princeton Monograph in Art and Archaeology, XXXIII). 
Hölscher, T. (1988) : « Historische Reliefs », in : Kaiser Augustus und die verlorene Republik, cat. exp., Mayence, Verlag Philipp van Zabern, p. 351-400.

Jucker, I. (1959) : « Ein Stirnziegel aus der Sammlung Johann Jacob Bachofen », in : Museum Helveticum, XVI, p. 60-68.

Karwiese, S. (1968-1971) : « Der tote Attis », in : Jahreshefte des österreichischen Archäologischen Instituts, XLIX, p. 50-62.

Koerte, A. (1897) : « Kleinasiatische Studien II », in : Athenische Mitteilungen, XXII, p. 1-51.

Koerte, A. (1900) : « Kleinasiatische Studien VI », in : Athenische Mitteilungen, XXV, p. 398-444.

Lancel, S. / Carrié, J.-M. / Saumagne, Ch. (1979) : Byrsa I : Mission archéologique française à Carthage. Rapports préliminaires des fouilles, 1974-1976, Rome, École française de Rome, (Collection École française de Rome, XLI), p. 283-310.

Lawall, M. L. (2003) : « < In the Sanctuary of the Samothracian Gods >. Myth, Politics and Mystery Cult at Ilion », in : Cosmopoulos,M. B. (éd.) : Greek Mysteries. The Archaeology and Ritual of Ancient Secret Cults, Londres, Routledge, p. 79-111.

LIMC : Lexicon Iconographicum Mythologiae Classicae, Boardman, J. et al. (éd.), Zurich / Munich, Artemis, 1981 sqq.

Macridy-Bey, Th. / Picard, Ch. (1921) : «Attis d'un Métroon ( ?) de Cyzique », in : Bulletin de Correspondance Hellénique, XLV, p. 436-470.

Marquardt, N. (1995) : Pan in der hellenistischen und kaiserzeitlichen Plastik, Bonn, Habelt (Antiquitas, XXXIII).

Mattern, T. (2000) : « Der Magna-Mater-Tempel und die augusteische Architektur in Rom », in : MDAIR, CVII, p. 141-153.

MDAIR : Mitteilungen des Deutschen Archäologischen Instituts, Römische Abteilung, Mayence, von Zabern.

Pensabene, P. (1982) : « Nuove Indagini nell'area del tempio di Cibele sul Palatino », in : Bianchini, U. / Vermaseren, M. J. (éd.) : La Soteriologia dei Culti Orientali nell'Impero Romano, Leyde, Brill (Études préliminaires aux religions orientales dans l'Empire Romain, XCII), p. 68-108.

Pensabene, P. / Sanzi Di Mino, M. R. (1983) : Museo Nazionale Romane, Le terrecotte, III, I, Antefisse, Rome, De Luca.

Pensabene, P. (1988) : « Scavi nell'area del Tempio della Vittoria e del Santuario della Magna Mater sul Palatino ", in : Archeologia Laziale IX. Nono Incontro di Studio del Comitato per l'archeologia Laziale, Rome, Consiglio Nazionale delle ricerche (Quaderni del Centro di Studio per l'archeologia Etrusco-Italica, XVI), p. 54-67.

Pensabene, P. (1998) : «Vent'anni di studi e scavi dell'Università di Roma « La Sapienza » nell'area Sud Ovest del Palatino (1977-1997) », in : Ciavarini, C. (éd.) : Il Palatino. Area Sacra sudovest e Domus Tiberiana, Rome, L'Erma di Bretschneider, p. 1-154.

Pensabene, P. (2002) : « Venticinque anni di ricerche sul Palatino : i santuari e il sistema sostruttivo dell'area sud-ovest », in : Archeologia Classica, LIII, p. 65-163.

Perrisin-Faberet, A. (2004) : Isis en Occident. Deuxième congrès international des études isiaques, table ronde Lyon 2002, Bricault, L. (éd.), Leyde, Brill.

Price, S. R. F. (1984) : Rituals and Power, The Roman Imperial Cult in Asia Minor, Cambridge, Cambridge University Press. 
Rieger, A.-K. (2004) : Heiligtümer in Ostia, Munich, Verlag Dr. Friedrich Pfeil (Studien zur antiken Stadt, VIII).

Rivera, R. R. (1993) : «Collegium dendrophorum : Corporacion profesional e cofradia metroaca », in : Gerion, XI, p. 175-183.

Rives, J. B. (1995) : Religion and Authority in Roman Carthago from Augustus to Augustine, Oxford, Clarendon Press.

Roller, L. E. (1999) : In Search of God the Mother. The Cult of Anatolian Cybele, Berkeley, University of California Press.

Romanelli, P. (1963) : « Lo scavo al Tempio della Magna Mater sul Palatino e nelle sue adiacenze », in : Monumenti Antichi, XLVI, p. 201-330.

Salis, A. von (1913) : « Die Göttermutter des Agorakritos », in : Jahrbuch des Deutschen Archäologischen Instituts, XXVIII, p. 1-20.

Schmidt, E. (1919) : Kultübertragungen, Giessen, Töpelmann (Religionsgeschichtliche Versuche und Vorarbeiten, VIII, 2).

Steuernagel, D. (2001) : « Kult und Community. Sacella in den Insulae von Ostia », in : MDAIR, CVIII, p. 41-56.

Steuernagel, D. (2004) : Kult und Alltag in römischen Hafenstädten, Stuttgart, Franz Steiner Verlag (Postdamer Altertumswissenschaftliche Beiträge, XI).

Töpperwein, E. (1976) : Terrakotten von Pergamon, Berlin / New York, De Gruyter (Pergamenische Forschungen, III).

Tomei, A. M. (1997) : Museo Palatino, Milan, Electra.

Vermaseren, M. J. (1977) : Cybele and Attis. The Myth and the Cult, Londres, Thames and Hudson.

Waelkens, M. (1986) : « The Imperial Sanctuary at Pessinus. Archaeological, Epigraphical and Numismatic Evidence for its Date and Identification », in : Zeitschrift für Epigraphik und historische Geographie Anatoliens, VII, p. 37-73.

Wiseman, T. P. (1984) : « Cybele, Virgil and Augustus », in : Woodman, T. / West,D. (éd.) : Poetry and Politics in the Age of Augustus, Cambridge, Cambridge University Press.

Wissowa, G. (1912) : Religion und Kultus der Römer, $2^{\mathrm{e}}$ éd., Munich, Beck'sche Verlagsbuchhandlung (Handbuch der Altertumswissenschaften, V, 4).

Wuilleumier, P. (1953) : Lyon. Métropole des Gaules, Paris, Les Belles Lettres.

\section{NOTES}

1. Pour le rayonnement du culte en province, cf. en général Vermaseren (1977), p. 126-144 et Beard / North / Price (1998), p. 337 sq.

2. Sur la dépendance entre Rome et Ostie, voir Rieger (2004), p. 261-263 et Steuernagel (2004), p. 258.

3. Pour la compilation des sources antiques, voir Graillot (1912), p. 25-69; Wissowa (1912), p. 317-327 et Schmidt (1919), p. 1, nº 1 ; voir, pour la discussion, Gruen (1990), p. 5-33.

4. Par ex. chez Cumont (1905), chap. III, p. 43-68 ; Steuernagel (2004), chap. V, p. 210-257 utilise le terme d' " étrangère "; Anne Perrisin-Faberet celui de "quasi-officiel », in Perrisin-Faberet (2004), p. 453. 
5. Sur la diffusion en grand Grèce à l'époque pré-hellénistique, voir Roller (1999), p. 119-234 ; Cf. Borgeaud (1996), p.17, pour la statue de culte de l'Agorakritos d'Athènes, qui devient déterminante ; voir aussi Tomei (1997), p. 148 sur les rapports entre la statue de Magna Mater du Palatin (ici, cf. fig. 2) et les œuvres attiques et pergaméniennes.

6. Voir Liv. 29, 11, 7 (... sacrum lapidem...). Diod. 33, 34, 1-3 (...тஷ் i $\varepsilon \rho \dot{\alpha . . .) . ~}$

7. Voir à ce sujet Roller (1999), p. 264-285 ; Coarelli (1988), p. 635-637.

8. Voir la compilation et l'exploitation des sources plus tardives chez Roller (1999), p. 264-271, qui à la différence de Borgeaud (1996), note 5, p. 78 sq., se prononce en faveur d'un lieu de provenance « double ».

9. Voir Roller (1999), p. 269.

10. À propos de la construction d'un passé mythique dès l'époque hellénistique et de son renouvellement à l'époque augustéenne, voir Roller (1999), p. 270 et note 43.

11. Ce nom est habituel à partir de la période hellénistique. Roller (1999), p. 67, 198 sq., 270 et notes 41-43, présente les sources littéraires impériales, que nous ne répéterons pas ici ; cf. aussi Wiseman (1984), p. 117-128 sur le rôle de Magna Mater dans l'Énéide. Comme exemples de l'utilisation du nom, nous renvoyons à : CCCA III, 1977, p. 68 sq., nº 258 ( $\mathrm{I}^{\mathrm{er}}$ siècle ap. J.-C.) ; CIL XIV suppl. I 4303 (III ${ }^{\mathrm{e}}$ siècle ap. J.-C.). Énée, né selon Hom. Il. 2, 820 sq. dans le mont Ida, devient l'ancêtre masculin des Romains par l'intermédiaire des rois d'Alba Longa.

12. Ceci est déduit a posteriori de Dion. Hal. 2, 19, 3-5.

13. L'interdiction pour les citoyens romains d'assumer la prêtrise telle que signalée chez Dion. Hal., loc. cit., note précédente, amène à se demander si l'on ne pouvait pas néanmoins exercer la fonction moyennant un renoncement à la citoyenneté. En outre, d'autres non-Romains, pas obligatoirement phrygiens, pourraient l'avoir exercée.

14. Sur le prêtre phrygien Battakes, voir Diod., 36, 13. Des preuves de l'apparition réglementée en public ont été conservées pour la fin de la période républicaine ; cf. aussi Dion. Hal., ant. 2, 19 ; Cic., leg., 2, 9, 22: 16, 40 ; Ov., fast., 4, 350.

15. Sur les transformations du paysage religieux de Rome, voir par ex. Beard / North / Price (1998), p. 245-301. Les représentations ne montrent au moins pas de castration totale.

16. CIL XIII 1751 (Lyon); CIL X 3698 (Puteoli) : occabus et corona comme insignes. Chez Polybe 21, 6,7 et 37,5 , ce sont des « figures et des pectoraux » qui sont mentionnés ; chez Dion. Hal., ant., 2, 19, 3-5 des « figures sur la poitrine ».

17. CCCA III, 1977, p. 68 sq., n 258 et CIL VI 2257.

18. Ajoutons deux pièces non citées ici, l'autel de L. Valerius Fyrmus CCCA III, 1977, p. 133 sq., $\mathrm{n}^{\circ} 422$ (II ${ }^{\mathrm{e}}$ siècle ap. J.-C.) (anaxyrides) et le buste CCCA III, 1977, p. 265 sq., n 250 (III ${ }^{\mathrm{e}}$ siècle ap. J.C.) (torques, coiffe, pectoral), tous deux à Rome. L'analyse de l'évolution des coutumes vestimentaires ne peut être effectuée ici. On constate toutefois que d'autres caractéristiques sont mentionnées dans les inscriptions (occabus et corona) que celles qui apparaissent dans les représentations (torques et anaxyrides).

19. Cf. note 16 .

20. Natascha Sojc, Kaiserlicher Palast und hegemoniale Struktur (thèse d'habilitation inédite), chap. II.2. : "Dersymbolische Wert architektonischer Form in republikanischer Tradition"; Pensabene (1998), p. 8-17 ; Cecamore (2002), p. 152-154 au sujet d'un lien possible de Magna Mater avec les Lupercalia. La terrasse du temple offrait une large vue sur le Tibre, sur lequel la déesse avait été transportée.

21. Voir plus loin le passage sur les ludi Megalensis. Selon Pensabene (1998), p. 36, la hauteur du podium atteignait près de $9 \mathrm{~m}$ (cf. aussi fig. 8-9).

22. Romanelli (1963), p. 221-240 ; Pensabene (1998) et Pensabene (2002), fig. 27 pour les substructions ; Mattern (2000).

23. Voir par ex. Lawall (2003), en part. p. 95-97. Il voit des liens entre le bâtiment aux mosaïques du sanctuaire est ( $3^{\mathrm{e}}$ quart du $\mathrm{III}{ }^{\mathrm{e}}$ siècle av. J.-C.) et les autres temples consacrés à Magna Mater. 
Des arguments comme le sol en mosaïque, la profondeur du vestibule et la grande taille de la base de la statue de culte n'ont cependant guère de pertinence ; cf. aussi Calza (1947), p. 188 sq.

24. Songeons aux sanctuaires de Palazzolo-Akrai, d'Éphèse ou de Priène; voir les brèves descriptions dans CCCA I, 1987, n 612-631, p. 184-189 (Éphèse) ; n 688-690, p. 203 sq. (Priène) ; CCCA IV, 1978, n 152-165, p. 61-66 (Akrai).

25. Les Megalesia sont mentionnées chez Varron, ling., 6, 15 ; Liv. 29-14,14 ; Cic., harus. resp., 24 affirme que comme les ludi Apollinares, les Megalesia avaient lieu devant le temple (Liv. 40,51,3) ; parmi les autres ludi importants, il y a par ex. les ludi Romani, cf. aussi Wissowa (1912), p. 460-464.

26. Romanelli (1963), p. 260-290 (p. 261 pour la datation) et p. 321-330 (pièces d'époque impériale) ; Pensabene (1982), p. 85-90, en part. p. 85 sq. (ont été trouvées dans le remplissage pré-augustéen du podium) ; cf. aussi Roller (1999), p. 274-279.

27. Voir CCCA IV, 1978, n 113-115, p. 44-46 (Tarente) ; n 130-132, p. 52 (Rhegium) ; CCCA II, 1982, p. 3-68, en part. p. 27, n $70 ;$ p. 43 sq., n 157-158 (Athènes, cf. aussi Salis [1913] pour la statue d'Attis dans le Métroon); p. 68-97, en part. p. 93 sq., n 308 (Pirée, cf. aussi Garland [1987], p. 129-131 pour les $\left.{ }_{20}^{20} A \tau \tau 1 \delta \varepsilon l \alpha\right)$ ) p. 549 (Amphipolis); p. 177-179 (Lesbos); LIMC III, 1986, cf. «Attis » (Maarten Vermaseren), p. 22-44, en part. p. 44.

28. Voir chez Roller (1999), fig. 70 ; Töpperwein (1976), p. 87 sq. et p. 227, nº 390 (Éros en tant qu'Attis), n 391 (bras d'un Attis ?) avec fig. 55.

29. Voir Roller (1999), p. 279.

30. Voir Rieger (2004), p. 154-157.

31. Seuls les prêtres apparaissant à cette époque utilisent des éléments vestimentaires non romains, cf. ci-avant.

32. Voir sur la diffusion de dendrophores Rivera (1993), en part. p. 176 sq. et note 6 ; sur les cannophores cf. ci-après, note 39.

33. Voir Beard / North / Price (1998), p. 336-338. L'influence des quindecemviri sur les collegia et les prêtres est attestée à ces différents endroits : CIL V, 4400 (Brixia) ; CIL X, 129 (Potentia) ; 4726 (Forum Popilii) ; 3698-3699 (Cumae / Puteoli) ; CIL IX, 981 (Compsa) ; 3764 (Suessula) ; CIL XII, 1567 (Dea Vocontiorum) ; CIL XIII, 1751 (Lyon) ; à Rome même : CIL VI, 29691 ; 1995.

34. Pour de bonnes descriptions des changements sociaux, cf.: Beard / North / Price (1998), p. 358 sq. ; Steuernagel (2001), en part. p. 54 sq. ; Rieger (2004), p. 26-29.

35. CIL XII, 409 (Marseille); Wuilleumier (1953), p. 89, pl. 10,2 et CCCA V, 1986, p. 133 sq., $\mathrm{n}^{\circ} 386$ (Lyon) : ...vires excepit et a Vaticano trans / tulit... ; CCCA VI, 1989, p. 14 sq. ; n 49 (Mayence-Kastel), dont le sanctuaire de Bellone in monte Vaticano demande à être rénové ; sur la relation entre Magna Mater et Bellone, cf. Rieger (2004), p. 138 sq. et Fishwick (1967), p. 142-160.

36. Voir le résumé de Rieger (2004), p. 119-122, fig. 90 ; sinon, Steuernagel (2004), p. 77.

37. CIL XIV, 324 ; sur le sanctuaire, cf. aussi Rieger (2004), p. 96, 123 sq.

38. Par ex. CIL XIV, 281 ; cf. Rieger (2004), p. 171.

39. À propos des hastiferi d'Ostie, cf. Rieger (2004), p. 269, MMB 66, 68-70; CIL VI, 2232 (consécration d'une hasta) de Rome. Les hastiferi sont encore mentionnés dans des inscriptions de Vienne, Mayence et Cologne ; cf. Fishwick (1967), p. 193 sq. - Les cannofori peuvent aussi être dans une certaine mesure considérés comme un phénomène spécifique à Ostie, où ils sont très fréquents. Ils sont attestés à Rome, Ostie, Mediolanum, Locres et Saepinum ; cf. Fishwick (1966), en part. p. 198.

40. On trouve par ex. une description chez Ov., fast., 4, 260 ; cf. aussi la représentation du récit sur un autel des musées capitolins CCCA III, 1977, p. 45 sq., n² 218, pl. 130.

41. Probablement y avait-il à la place du futur Piazzale une halte pour Magna Mater, signalée par Ovide (fast., 4, 291 (atria tibertina)), cf. Rieger (2004), p. 247-249; sur les antéfixes, cf. Jucker (1959) ; Hölscher (1988), en part. p. 379 sq., n 211 du Palatin ; Pensabene / Sanzi Di Mino (1983), n $966-973$; CCCA III, 1977, p. 10, nº 11 pour l'exemplaire du Palatin ; p. 38, n 202-203 pour celui du Forum ; p. 135-138, n 427 et 432-435 pour celui du Piazzale delle Corporazioni ; p. 124, n 397 ; 
p. 136, $\mathrm{n}^{\circ}$ 428-430 est probablement l'exemplaire du sanctuaire de Magna Mater à Ostie (v. Rieger [2004], p. 248 et note 1203).

42. Voir Steuernagel (2004), p. 21 sq., 222 et 240 , et le compte rendu de A.-K. Rieger à propos de Steuernagel (2004) in : Göttinger Forum für Altertumswissenschaften, IX, 2006, p. 1113-1125, en part. p. 1122 et note 18. Quatre lampes sont mentionnées dans CCCA III, 1977 sous les $n^{\circ} 437-440$ (p. 138 sq.).

43. Tomei (1997), p. 148, cat. 128 ; cf. aussi Bartolli (1947).

44. Cf. plus haut.

45. Pensabene (1982), p. 98 ; sur Carthage, cf. CCCA V, 1986, p. 36 sq., n 98. James B. Rives (Rives [1995], p. 75) note la rareté du nom de Fonteius en Afrique et son absence dans la Proconsularis.

46. Voir Lancel / Carrié / Saumagne (1979).

47. Voir Becatti (1961), vol. 1, p. 71, n 99.

48. Cf. ci-avant.

49. On en sait peu sur le Métroon de Cyzique, cf. Macridy-Bey / Picard (1921), en part. p. 436-443 ; Devambez (1937), p. 178-194. Deux des pièces se trouvent à Istanbul, une à Liverpool. Sur la datation stylistique, cf. Devambez (1937), p.187-194 (E. 2./déb. III siècle ap. J.-C.-); Nathalie Marquardt ([1995], p. 109 sq.), qui se montre plutôt adversaire d'une association des figures.

50. La question de la datation exacte des sculptures conditionne évidemment l'ordre de succession du modèle et de l'imitation.

51. Voir Calza (1947), p. 186, 197 sq. ; Rieger (2004), p. 153 sq. ; Steuernagel (2004), p. 233, 235 ; Karwiese (1968-1971), en part. p. 60 sq., fig. 8.

52. Voir la description chez Devreker/ Waelkens (1984), p.13-20. On trouve aussi une description chez Waelkens (1986), p.39-47. Cf. p. 48-60 à propos de la datation à l'époque tibérienne au moyen de l'ornementation architecturale (cf. aussi Hugo Thoen in: Devreker / Waelkens [1984], p.142-160); sur le complexe d'escalier et le théâtre, cf. Waelkens (1986), p. 43 sq., p. 60-67 avec des exemples de comparaison plus ou moins utilisables; cf. aussi Devreker / Thoen / Vermeulen (1995).

53. Waelkens (1986), p. 38 sq. à propos des vestiges des $V^{e}$ et $I^{e}$ siècles av. J.-C. dans l'aire des temples plus tardive; p. 67-72, en part. p. 69 (y compris la citation) sur l'identification en tant que temple du culte impérial ; cf. aussi Devreker / Waelkens (1984), p.20, où la pauvreté des arguments en faveur du culte impérial est signalée : «Le seul [...] indice est la présence du petit théâtre, un phénomène typiquement occidental.» Voir aussi Devreker / Thoen / Vermeulen (1995); Waelkens (1986), p. 67, note 211 cite l'explication de Pieter Lambrechts, selon laquelle Magna Mater et les Divi étaient adorés conjointement dans le temple; cf. aussi Price (1984), p. 168 , note 93 ; p. 268 , cat. $n^{\circ} 112$, qui se prononce contre l'hypothèse du culte impérial dans ce temple.

54. Voir CCCA I, 1987, p. 25-27, n 59 et 60 ; Koerte (1897), en part. p. 38-43, n 23 et Koerte (1900), p. 437-439, n 63 ; cf. aussi Devreker / Waelkens (1984), p. 20.

55. Voir Herodian I, II, 2 ; Graillot (1912), p. 348 (fleuve sacré).

56. Voir ci-avant. Des mystères liés à Magna Mater (CCCA I, 1987, p. 26 sq., $n^{\circ}$ 60) et les theai $(\theta$ Éal) consacrées par le prêtre impérial (cf. Waelkens [1986], p. 70 et note 230) sont attestés à Pessinonte.

57. Les exemples italiens de la période hellénistique sont suffisamment connus. Voir Coarelli (1987). Comme parallèle en Asie mineure, on peut nommer le théâtre de l'Esculapium, à Pergame (cf. Waelkens [1986], p.67), mais il n'existe en aucune façon de parenté architecturale étroite entre le théâtre et le temple. Voir Hanson (1959). 
INDEX

Mots-clés : Magna Mater, Rome

Schlüsselwörter : Magna Mater, Rom

\section{AUTEURS}

\section{ANNA-KATHARINA RIEGER}

Anna-Katharina Rieger travaille en collaboration avec le centre interdisciplinaire de recherche « Différence et intégration » de la Martin-Luther-Universität à Halle-Wittenberg. Pour plus d'informations, voir la notice suivante. 